\title{
Coherence measurements in synthetic turbulent boundary layers
}

\author{
By OMER SAVAŞ \\ Schơl of Aerospace, Mechanical, and Nuclear Engineering, University of Oklahoma,
} Norman, Oklahoma 73019

\section{AND DONALD COLES}

Graduate Aeronautical Laboratories, California Institute of Technology, Pasadena, California 91125

(Received 11 September 1984 and in revised form 15 May 1985)

Synthetic turbulent boundary layers were constructed on a flat plate by generating systematic moving patterns of turbulent spots in a laminar flow. The experiments were carried out in a wind tunnel at a Reynolds number based on plate length of $1.7 \times 10^{6}$. Spots were generated periodically in space and time near the leading edge to form a regular hexagonal pattern. The disturbance mechanism was a camshaft that displaced small pins momentarily into the laminar flow at frequencies up to $80 \mathrm{~Hz}$. The main instrumentation was a rake of 24 single hot wires placed across the flow in a line parallel to the surface. The main measured variable was local intermittency; i.e. the probability of observing turbulent flow at a particular point in space and time. The results are reported in numerous $(x, z, t)$-diagrams showing the evolution of various synthetic flows along the plate. The dimensionless celerity or phase velocity of the large eddies was found to be very nearly 0.88 , independent of eddy scale. All patterns with sufficiently small scales eventually showed loss of coherence as they moved downstream. A novel phenomenon called eddy transposition was observed in several flows that contained appreciable laminar regions. The original large eddies were replaced by new eddies at new positions, intermediate to the original ones, while preserving the hexagonal pattern. The present results, together with some empirical properties of a turbulent spot, were used to estimate the best choice of scales for constructing a synthetic boundary layer suitable for detailed study as a model for a natural flow. The values recommended are: spanwise period/thickness $\approx 2.5$, streamwise period/thickness $\approx \mathbf{8 . 0}$.

\section{Introduction}

The current view of turbulent fluid motion is that turbulent shear flows are not entirely random, but contain organized eddy structures that represent characteristic concentrations of vorticity at the largest lateral scale of the flow. A comprehensive review of the subject of organized motion in turbulent flows has been published by Cantwell (1981).

Any attempt to find and study such organized motions in the turbulent boundary layer is confronted by serious difficulties. The eddies that make up the turbulent boundary layer are three-dimensional, and they occur in various stages of development at random places and times. Presumably, the eddies have a typical signature in terms (say) of surface pressure, surface friction, local turbulence intensity, interface 
geometry, large-scale vorticity, and the like. However, this signature cannot be determined until the eddy has been found, and the eddy cannot readily be found unless its signature is known. Another difficulty is that most measurements of structure in boundary layers have been made using single fixed probes. Such measurements are not well suited to describing the properties of an irregular pattern of three-dimensional large eddies being convected past the probe because no information is available about the lateral position of these eddies. Hence, their properties cannot be sharply defined.

As a possible means of bypassing these difficulties, Coles \& Barker (1975) proposed the concept of a synthetic turbulent boundary layer and made some measurements in one such flow. Their point of departure was the fact that natural transition from laminar to turbulent flow in a boundary layer is characterized by the appearance of turbulent spots. The turbulent spot, which was discovered by Emmons (1951) and was first documented experimentally by Schubauer \& Klebanoff (1955), is an arrowhead-shaped region of turbulence that appears in a laminar boundary layer (when there is a suitable natural or artificial local disturbance) and moves downstream, growing in size nearly linearly in all directions. The ensemble-averaged structure of the spot was identified as essentially a large horseshoe vortex by the work of Coles \& Barker (1975), Wygnanski, Sokolov \& Friedman (1976), and Cantwell, Coles \& Dimotakis (1978). Consequently, in the context of the coherent-structure concept, the spot suggests itself as a possible prototype large eddy for the turbulent boundary layer. Cantwell et al. end by viewing the spot not as a prototype large eddy but as an alternative flow to the boundary layer, primarily because of the eventual discrepancy in scale. There is by now considerable evidence that the structure of a single spot is quite different from the average picture constructed from ensemble-mean measurements. While a spot grows nearly linearly in all directions, its basic building blocks seem to be eddies whose streamwise dimensions are comparable with the local spot thickness (see, for example, Matsui 1979; Gad-el-Hak, Blackwelder \& Riley 1981 ; Wygnanski, Zilberman \& Haritonidis 1982; Itsweire \& Van Atta 1984).

Nevertheless, the isolated spot is expected to have structural features in common with characteristic large-scale vorticity concentrations in the turbulent boundary layer. Zilberman, Wygnanski \& Kaplan (1977) and Haritonidis, Kaplan \& Wygnanski (1977) followed an artificially generated spot into a turbulent boundary layer for very large distances. They found that the growth of the spot was severely inhibited in the streamwise and span wise directions, but not in the normal direction. The characteristic celerity of the spot in the boundary layer was about $0.9 u_{\infty}$. On the basis of these experiments, Wygnanski (1978) refers to the turbulent spot as an orderly structure that may be viewed as the basic module for the turbulent boundary layer.

The experiment carried out by Coles \& Barker was to generate a regular hexagonal pattern of turbulent spots in the laminar boundary layer near the leading edge of a flat plate, and to sample the flow farther downstream at times locked to the phase of the disturbance generator. They found that periodicity in space and time persisted downstream, and that the average velocity profile was close to that which would be expected in a natural turbulent boundary layer at the same Reynolds number. Figure 1 shows a photograph of a single spot in water, using surface dye for flow visualization, together with a photograph of a synthetic turbulent boundary layer under the same conditions in the same channel.

The present research continues the work begun by Coles \& Barker. Their measurements were made in water, using momentary jets to create turbulent spots and using a single-channel laser-Doppler velocimeter as main instrumentation. 


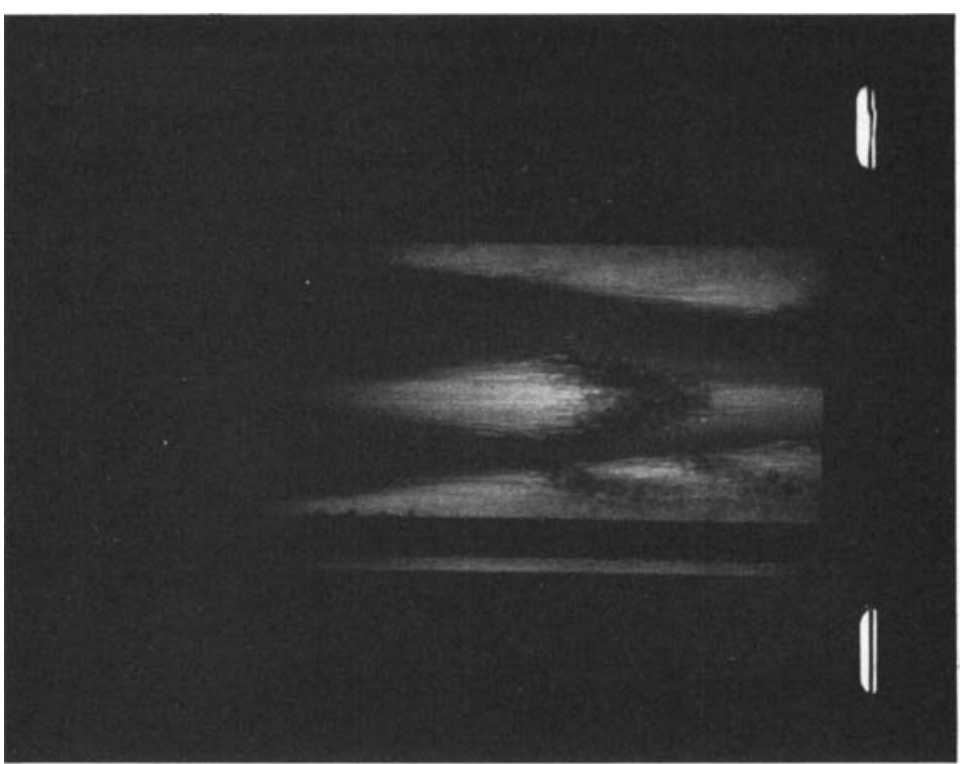

(a)

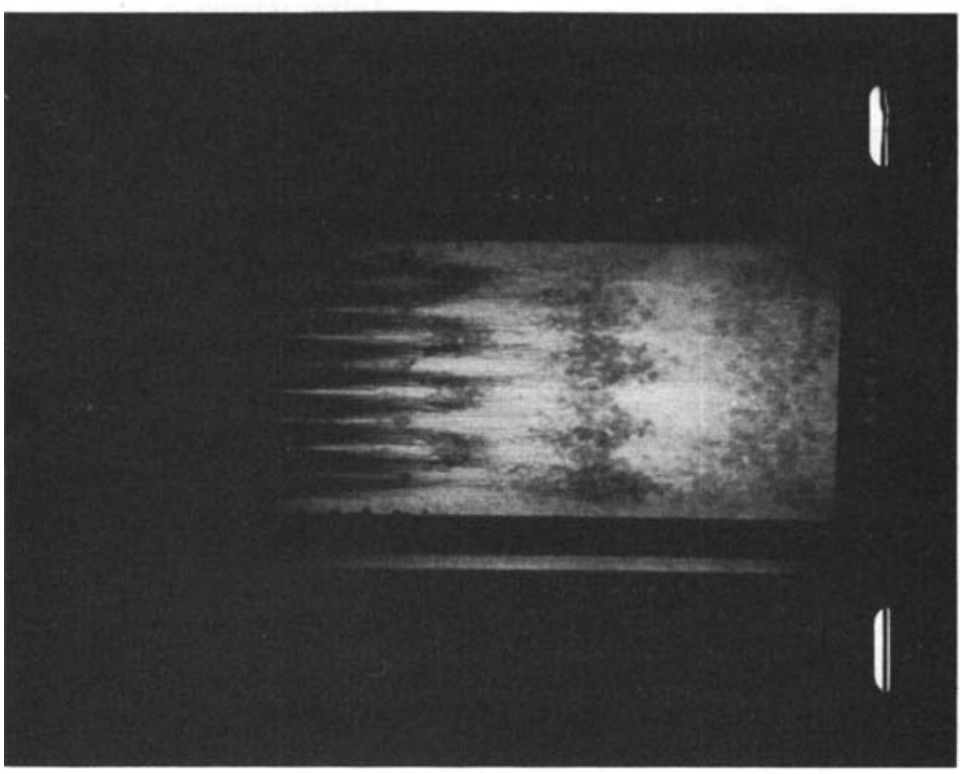

(b)

Figure 1. Dye visualization in water channel for flow studied by Coles \& Barker: $(a)$ turbulent spot, (b) synthetic turbulent boundary layer.

Because of an insufficient flow rate for the main pump and insufficient width of the channel the Reynolds number was relatively low, and the useful region of the plate surface was severely limited by transverse contamination from the sidewalls. There was also a substantial acceleration of the free stream along the plate. The present experiments were carried out under conditions that avoid these problems, particularly 
the problem of transverse contamination. The main difference is that the present measurements were made in a wind tunnel, thus requiring a shift to the hot-wire anemometer as the main instrumentation, and requiring also a shift to a disturbance mechanism capable of operating at much higher frequencies. The new apparatus and instrumentation are described in $\$ 2$.

As a practical matter, the research program now in progress at GALCIT (Graduate Aeronautical Laboratories, California Institute of Technology) has separated the problem of coherent structure in the turbulent boundary layer into two problems, one dealing with the term 'coherent', and the other with the term 'structure'. Only work on the first problem is reported here, in $\$ 3$ and 4 . The basic premise is that the most legible signature of a large eddy is the presence of a large turbulent bulge in the interface between the boundary layer and the free stream. The emphasis is on pattern and scale, as revealed by measurements of intermittency in the outer part of the synthetic layer. An appreciable range of streamwise and spanwise scales has been explored in an attempt to identify at least one synthetic flow that is optimum in the sense of having close eddy spacing and having also strong coherence over a large fraction of the plate surface. The flow that in our opinion best meets this specification is depicted in figure $11(a)$ and is described quantitatively in $\S 4.4$.

Except for one conjecture about the role of the turbulent spot as a prototype large eddy for the turbulent boundary layer, the discussion in $\$ 4$ deliberately avoids the subject of structure. At this stage we do not know whether or not various mean properties of the recommended synthetic flow coincide, over a reasonable range of Reynolds numbers and to practical accuracy, with the corresponding mean properties of a carefully tripped turbulent boundary layer. Some independent evidence on this question is available in the references cited at the end of $\$ 4.3$. What is ultimately required to resolve the question of structure, however, is information about the large-scale mean velocity, vorticity, intermittency, and turbulence fields at constant phase in the recommended synthetic flow. Detailed measurements for this purpose are now being carried out.

The present paper is based on the doctoral thesis of Savaş $(1979 a)$, parts of which have been briefly reported in two conference proceedings (Savaş $1979 b$ and Coles \& Savaş 1979).

\section{Experimental arrangements}

\subsection{Model and flow conditions}

Throughout this paper a right-hand rectangular coordinate system, oriented as shown in figure 2 , is used. The streamwise coordinate $x$ is measured from the leading edge of the plate; the normal coordinate $y$ is measured from the plate surface; and the spanwise coordinate $z$ is measured from the plate centreline.

The flat-plate model in figure 2 is made from two sections of aluminium-alloy plate. The front section is $30.5 \mathrm{~cm}$ long, $114.0 \mathrm{~cm}$ wide and $1.9 \mathrm{~cm}$ thick. The rear section is $233.7 \mathrm{~cm}$ long and $1.27 \mathrm{~cm}$ thick; the width increases from $114.0 \mathrm{~cm}$ at the joint to $117.2 \mathrm{~cm}$ at the trailing edge. The leading-edge contour is a 10:1 ellipse to avoid large positive pressure gradients which could cause separation and/or premature transition near the leading edge. The working surface of the assembled model is smoothed and polished.

The experiments were carried out in the Merrill wind tunnel at GALCIT. The leading edge was at the beginning of the test section, which was partitioned by the 


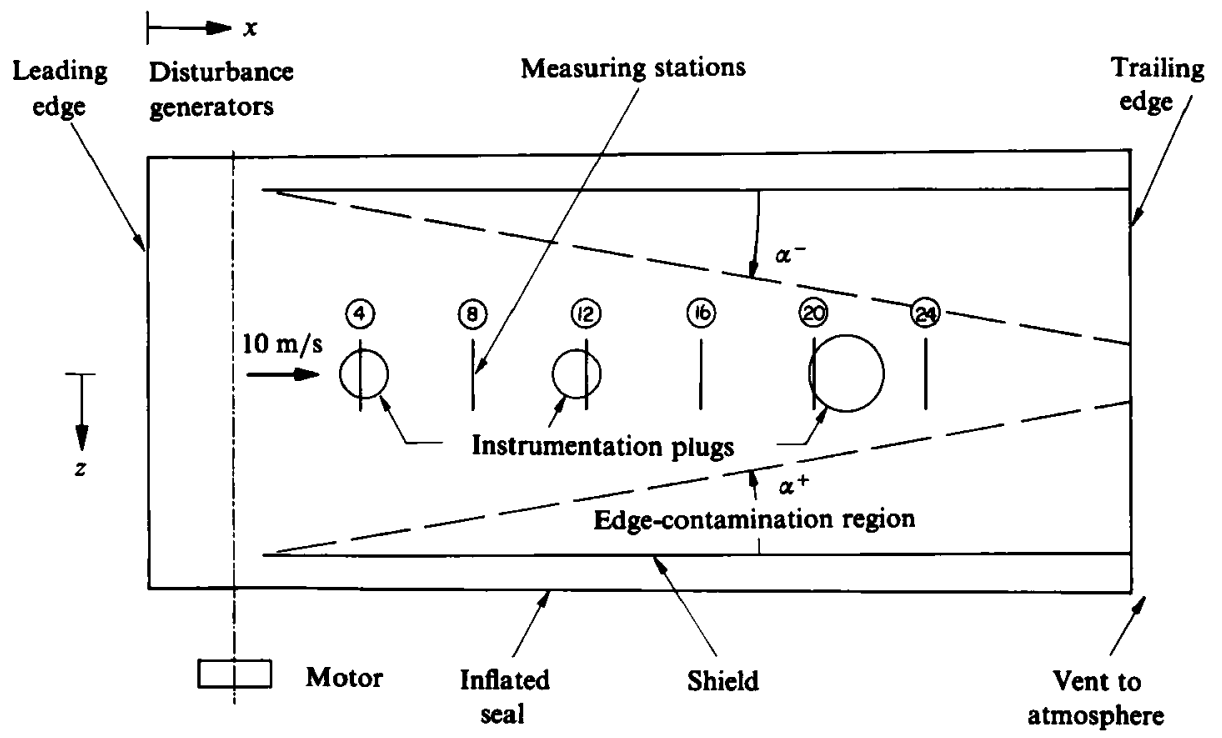

Figure 2. Plan of flat-plate model. Experimentally determined boundaries of transversecontamination regions are shown as dashed lines. For coordinates of measuring stations 4-24, see table 1.

flat-plate model along its entire length. The plate was supported from below by six streamlined struts. Narrow gaps between the plate and the sidewalls of the tunnel were sealed by pressurized latex tubing. Various flow-control devices were used to obtain a good approximation to uniform flow. These included:

(1) Six individually adjustable supports capable of bending the plate substantially.

(2) Two extruded-angle metal shields extending from $x=31 \mathrm{~cm}$ to $275 \mathrm{~cm}$ at $z= \pm 48.3 \mathrm{~cm}$. These shields are $12.7 \mathrm{~cm}$ high and are intended to isolate the flow over the plate from the boundary layers developing on the tunnel sidewalls. The shields are also used to locate and support a hot-wire rake.

(3) A two-dimensional blister to modify the ceiling contour of the tunnel at the entrance to the test section. The maximum thickness of the blister is about $0.8 \mathrm{~cm}$ at $x \approx 20 \mathrm{~cm}$.

(4) A screen of $20 \%$ solidity across the upper half of the test section at the trailing edge of the plate, just before the peripheral vent to atmosphere. The screen matches the overall pressure loss in the upper flow channel to the pressure loss caused by the presence of the supporting struts and other excrescences below the plate. The test-section pressure at a speed of $10 \mathrm{~m} / \mathrm{s}$ is about $0.08 \mathrm{mmHg}$ above atmospheric pressure.

The measured final pressure coefficient is constant to within \pm 0.01 over the useful part of the working surface. The presence of the rake has no significant upstream effect on the pressure distribution. With no artificial disturbances, the boundary layer on the plate centreline is laminar along the entire length of the plate $\left(R e=1.7 \times 10^{6}\right.$ at the trailing edge at $10 \mathrm{~m} / \mathrm{s}$ ). The edge-contamination regions emanating from the two boundary-layer shields were mapped by a surface tube and found to grow linearly at the accepted angle of about $10^{\circ}$. The edges of the contaminated regions are indicated in figure 2 by the dashed lines. 

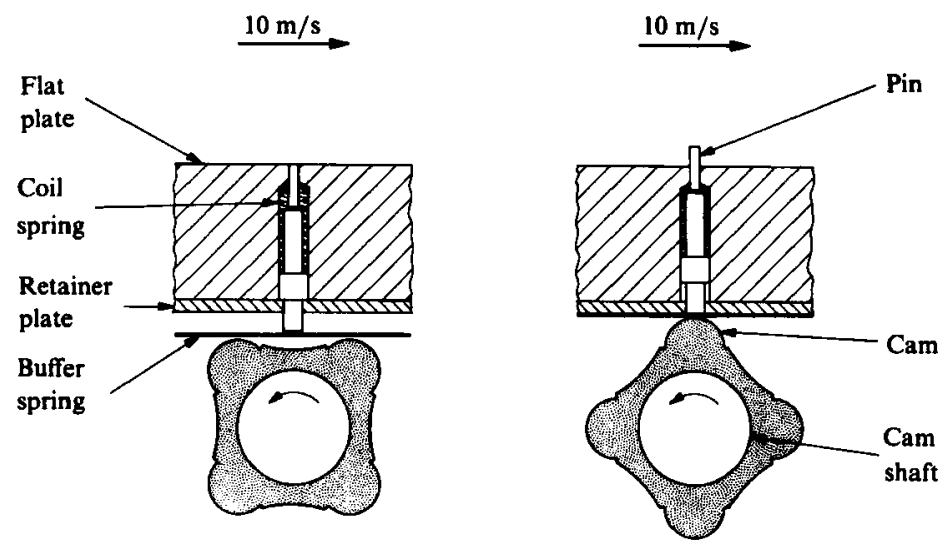

Fiqu Re 3. Detail of disturbance-generating mechanism. Left: pin retracted. Right: pin displaced into boundary layer by cam.

\subsection{Disturbance-generating mechanism}

Disturbances at the high frequencies required when working in air rather than water were generated mechanically at $x=22.9 \mathrm{~cm}$ by momentarily displacing small cylindrical pins into the laminar boundary layer. Figure 3 shows some geometrical details of the mechanism. The basic element is a nylon pin embedded in the front section of the plate. A compression spring keeps the pin retracted and in contact with a retainer plate so that the plate surface is normally uninterrupted. The pin is periodically displaced into the boundary layer by a rotating cam made from nylon impregnated with molybdenum disulfide. A cantilevered beryllium-copper leaf spring between each pin and its associated cam ensures non-destructive contact between the two moving elements. The support geometry and the length and thickness of the leaf spring were varied until the action, as observed with stroboscopic lighting, was smooth and free of resonance at all cam speeds of interest. Fatigue failure of the spring material was prevented by providing a cooling air flow over the mechanism.

The camshaft is mounted on the lower side of the front section of the plate. It is supported at seven locations on needle bearings and is shielded from the air flow by a curved shield which extends from sidewall to sidewall. One end of the camshaft extends out of the wind tunnel and is connected to an electric motor by a flexible coupling. The phase-locked-loop servo system developed by Cantwell (1975; see also Cantwell \& Coles 1983) was modified to drive the camshaft. In the present application, the feedback signal originates at a 200 -line optical encoder on the motor shaft.

The camshaft is fitted with six long nylon cam sleeves separated by short needle bearings. The sleeves are carefully aligned, with an individual four-lobe cam corresponding to each pin in the plate. Any cam lobes that are not wanted are machined off the sleeves. Normally two lobes $180^{\circ}$ apart are retained at each active pin location. Alternate active cams along the length of the camshaft are displaced by $90^{\circ}$. Thus the disturbance generator produces a hexagonal pattern in a $(z, t)$-plane when the shaft is rotating. The spacing $\zeta$ in the $z$-direction is fixed by the cam in use. The period $\tau$ in time is half the period of shaft rotation. For convenience, the frequency $f$, where $f \tau=1$, is sometimes specified instead of the corresponding period $\tau$. This frequency could be varied continuously from about $1 \mathrm{~Hz}$ to about $180 \mathrm{~Hz}$.

Altogether, 201 pins are installed in the model at a station $22.9 \mathrm{~cm}$ from the leading edge. The pins have a diameter of $0.102 \mathrm{~cm}$ and are spaced $0.508 \mathrm{~cm}$ apart. At several 
lateral positions two adjacent pins are omitted to make room for the needle bearings used to support the camshaft. The minimum uniform pin spacing is therefore $1.524 \mathrm{~cm}$. This spacing can be increased in increments of $0.508 \mathrm{~cm}$ up to the full width of the plate. With a hexagonal disturbance pattern, the minimum spanwise pitch or wavelength $\zeta$ is $3.048 \mathrm{~cm}$. The three values actually used in the present tests are 6.10 , 9.14 , and $12.19 \mathrm{~cm}$. There are slight inadvertent variations in cam radius and pin length, amounting to perhaps $\pm 0.005 \mathrm{~cm}$; these variations are sometimes detectable in the data as small variations in size and strength of the turbulent spots.

The disturbance mechanism was designed to work at any tunnel speed above about $10 \mathrm{~m} / \mathrm{s}$. Higher speed increases the Reynolds number at the disturbance station (i.e. increases the margin with respect to the small-disturbance stability boundary), and also thins the boundary layer, so that the pins seem to be larger and to extend farther into the flow. The normal pin displacement is $0.165 \mathrm{~cm}$, although it could be increased to perhaps $0.2 \mathrm{~cm}$ at the cost of a substantial increase in the loads on the cam mechanism. Tests demonstrated that at a tunnel speed of $10 \mathrm{~m} / \mathrm{s}$ spots were always generated when the pins were displaced into the flow at any frequency within the range of the disturbance mechanism. At a tunnel speed of $8 \mathrm{~m} / \mathrm{s}$ no spots were generated.

\subsection{Instrumentation}

Twenty-four commercial single hot-wire probes were mounted in a rake configuration transverse to the flow and parallel to the plate surface. The rake was suspended from a full-span bridge by three struts, as shown in figure 4 . All electrical wiring except for connections at the probes is contained within the rake, struts and bridge. The 24 probes have no common electrical connection. The entire rake and bridge structure is filled with epoxy resin for increased stiffness.

The 24 hot-wire probes are located symmetrically about the plate centre and are spaced $0.762 \mathrm{~cm}$ apart. The span of the rake is $17.5 \mathrm{~cm}$. The hot-wire sensors are parallel to the plate surface and hence respond primarily to the streamwise component of velocity. The sensors are $2.5 \mu \mathrm{m}$ in diameter and about $0.15 \mathrm{~cm}$ in length. They are made of platinum-plated tungsten wire and were operated at a constant temperature of $250^{\circ} \mathrm{C}$. No wire failure occurred during the experiments, which required several hundred hours of tunnel running time.

Twenty-four constant-temperature hot-wire anemometer circuits were fabricated for these experiments. Each circuit includes an intermittency meter consisting of a passive RC bandpass filter, a rectifier, a level detector, and a retriggerable monostable multivibrator, or one-shot. Each anemometer with its associated intermittency meter is contained on a single printed-circuit board. In the same enclosure is a digital multiplexer which allows the 24 bits of intermittency information to be latched at read time and later transferred to the computer in two 12-bit sections.

Intermittency is the primary measured variable in the present research. The basic assumption is that turbulent flow can be distinguished from laminar flow in terms of energy content at high frequencies. After some preliminary measurements, the cutoff frequencies of the bandpass filters were set at 2.7 and $7.0 \mathrm{kHz}$ and were left fixed throughout the course of the experiments. The threshold level on individual channels varied slightly with differences in the particular wires and components in the units. The pulse lengths of the one-shots were set to $1 \mathrm{~ms}$. No attempt was made to correct the data for the associated time lag at the end of a turbulent region.

Adjustment of the intermittency meters was essentially a subjective process. All 24 circuits were tuned in a turbulent boundary-layer flow generated by taping a 


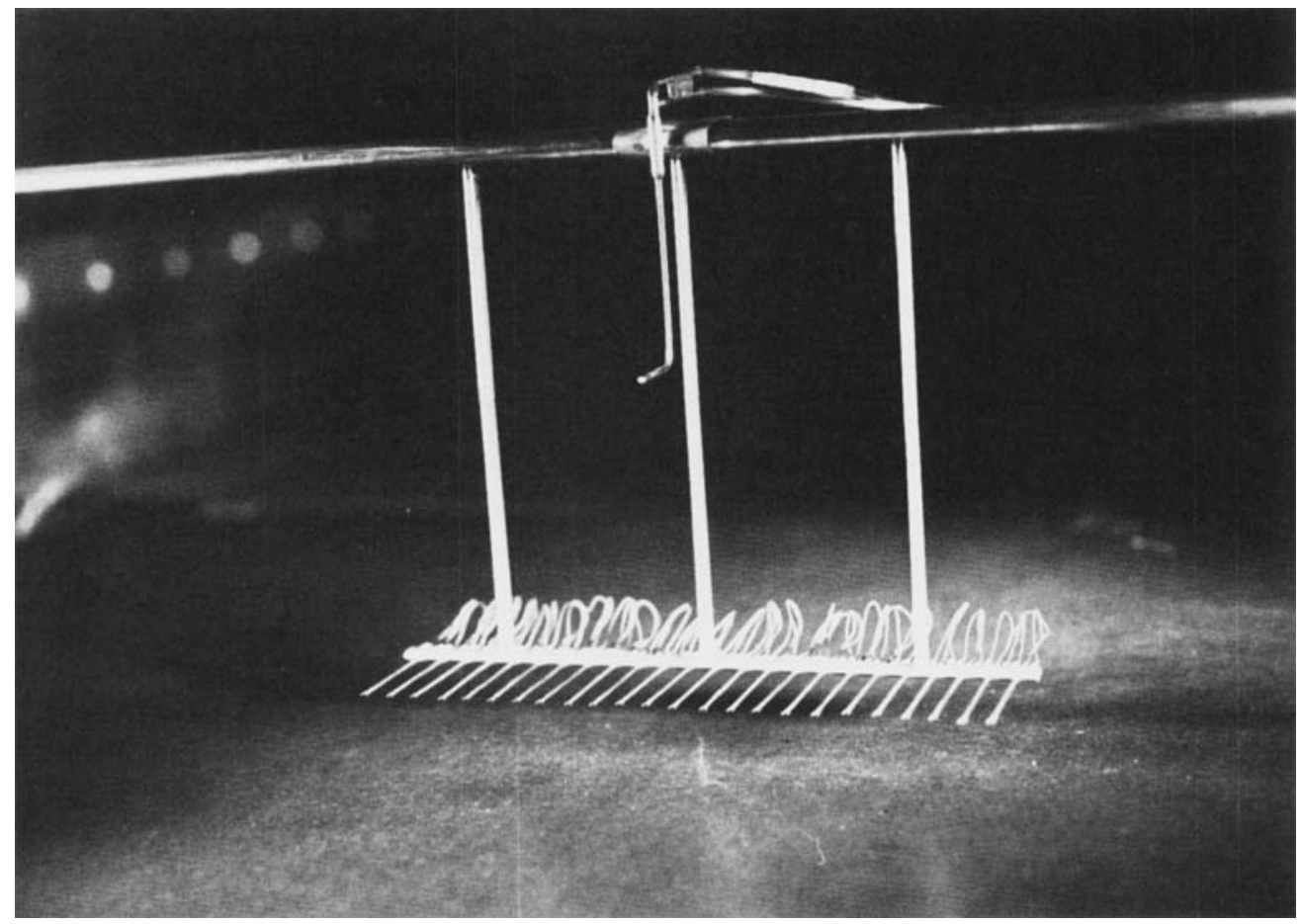

Figure 4. Close view of hot-wire rake.

serrated-tape trip onto the plate surface at $x=22.9 \mathrm{~cm}$ (slightly upstream of the pins, pins retracted). The trip extended from wall to wall. The rake was placed well downstream at a suitable distance from the plate surface. After considerable adjustment of probe height and circuit parameters, the average intermittency factor seen by the 24 wires at $x=118 \mathrm{~cm}, y=2.02 \mathrm{~cm}, U=10 \mathrm{~m} / \mathrm{s}$ was 0.50 , with extreme individual readings of $\mathbf{0 . 4 8}$ and $\mathbf{0 . 5 3}$. The intermittency function was also measured at a few other points across the tripped boundary layer. The data were entirely compatible with similar results previously reported in the literature (e.g. Klebanoff 1954).

The tunnel dynamic head $\frac{1}{2} \rho U^{2}$ was continuously monitored by a Pitot-static probe mounted from the tunnel ceiling. To measure the free-stream velocity $u_{\infty}$ near the hot wires, a second Pitot-static probe was installed on the hot-wire rake. The tip of this Pitot-static probe is about $8.7 \mathrm{~cm}$ above the sensors of the hot-wire probes. Finally, 44 surface-pressure taps are provided on the flat plate. Of these, 16 are on the centreline and 14 are on each of the lines $z= \pm 30.5 \mathrm{~cm}$.

\subsection{Data acquisition}

The data-acquisition system used for the experiments includes a preset counter which serves as a master clock. The output is a $2-\mathbf{M H z}$ crystal-controlled pulse train that can be divided by any desired integer $N$ (set on switches) to obtain a time base for the experiments. The number $N$ has a special significance for the present data. The pulse frequency $2000000 / N$ is again divided by 200 in the 200 -line encoder which controls the phase-locked camshaft drive. Hence, the period of the camshaft is $N / 10000 \mathrm{~s}$. Inasmuch as the nominal free-stream velocity in the present experiments is $10 \mathrm{~m} / \mathrm{s}$ or $10000 \mathrm{~mm} / \mathrm{s}$ (actually $10130 \mathrm{~mm} / \mathrm{s}$, on the average), the number $N$ is 

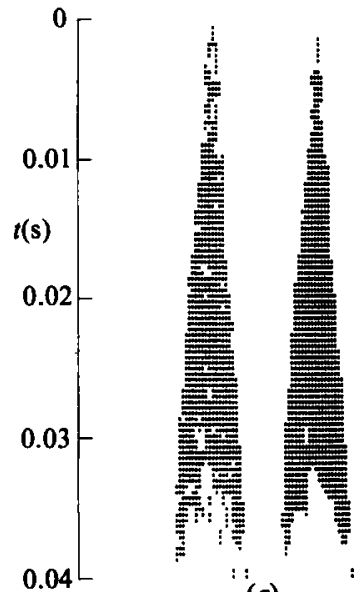

(a)

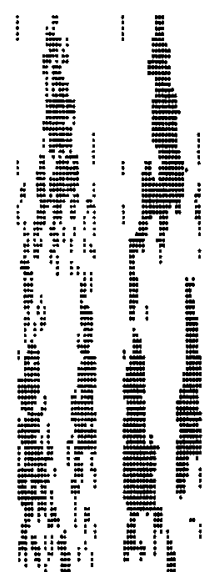

(b)

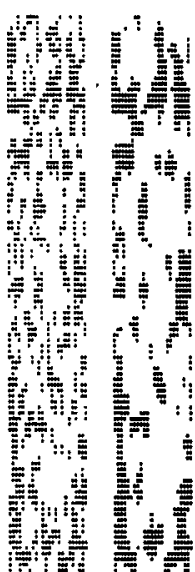

(c)

Fiaure 5. Typical unfiltered and filtered raw intermittency data for $U=10 \mathrm{~m} / \mathrm{s}, x=87.3 \mathrm{~cm}:(a)$ spot, $y=1.02 \mathrm{~cm}, N=800$ (run 538); (b) synthetic turbulent boundary layer, $y=1.36 \mathrm{~cm}, 12.2 \mathrm{~cm}$ cam, $N=800$ (run 117); (c) tripped turbulent boundary layer, $y=1.70 \mathrm{~cm}$.

for practical purposes the distance in millimeters travelled by the free-stream flow during one camshaft revolution, or during two cycles of the periodic disturbance pattern.

Intermittency data were recorded as a continuous time series on magnetic tape. A typical file contains one identification record and 400 data records, each corresponding to 5 camshaft revolutions. At each pulse of the time-base clock, five 16-bit words were read into the computer. The first two words are intermittency data. The remaining three words are digitized analog signals, the first being a general-purpose signal (used, for example, for surface-pressure measurements) and the other two the ceiling and rake dynamic-pressure readings.

\subsection{Digital filtering}

The intermittency data were crudely filtered before ensemble averages at constant phase were computed. There are two reasons: to sharpen the classification of the flow into laminar and turbulent regimes; and to provide a means for communication between adjacent wires, which sometimes have slightly different responses in the same flow. After some experimenting, the filtering method chosen was to centre a rectangular window, 3 wires wide and 5 samples long, over each bit of the pattern. If the sum of the 15 bits was 8 or more, the centre bit was replaced by a one; if the sum of the 15 bits was 7 or less, the centre bit was replaced by a zero. The data from wires 1 and 24 , at the ends of the rake, retained their original values. The same $3 \times 5$ filter window was used for all of the data processing. Because the sampling frequency varies inversely with $N$, the effect of filtering is uniform for different probe stations but not for different cam speeds. Figure 5 shows short segments of typical intermittency data, before and after filtering, for three flows. These are the turbulent spot, the synthetic boundary layer, and the natural boundary layer (i.e. the flow tripped by the serrated-tape trip).

The filtered data were ensemble averaged over each file as a $24 \times 200$ array, covering one camshaft revolution. In principle, the two cycles of a revolution should be identical and could be superimposed. In practice, however, slight variations in maximum pin displacement are detectable as variations in peak intermittency within 


\begin{tabular}{|c|c|c|}
\hline & & $x(\mathrm{~cm})$ \\
\hline Leading & edge & 0 \\
\hline Pins, tap & & 22.9 \\
\hline Station & 4 & 56.9 \\
\hline & 8 & 87.3 \\
\hline & 12 & 117.8 \\
\hline & 16 & 148.3 \\
\hline & 20 & 178.8 \\
\hline & 24 & 209.3 \\
\hline Trailing & edge & 264.2 \\
\hline
\end{tabular}

TABLE 1. Streamwise stations in $\mathrm{cm}$.

a turbulent region. For this reason the fundamental period of the experiments is sometimes taken as one revolution rather than one cycle.

Two characteristic values for overall intermittency were also calculated. One is the global mean intermittency; i.e. the value obtained by averaging over one revolution and over the number of wires corresponding to one spanwise period of the disturbance pattern. This value is usually close to the global mean value used to set the probe height (the target value was a global mean of 0.40 for $N=800$ ). The second characteristic overall intermittency is the median value, midway between the largest and smallest values occurring in the filtered and ensemble-averaged $24 \times 200$ array. This value identifies the contour whose interior is shaded in various figures below to indicate regions of turbulence. The median is preferable to the mean for this purpose because it does not decrease towards zero in flows with low inherent intermittency, especially low-frequency patterns in the upstream region of the flow.

\section{Scope of experiments and presentation}

The main parameters of the various synthetic flows studied during the present research are listed in several tables. Table 1 gives the distance from the leading edge of the plate to the pin station and to the six rake stations. Table 2 gives the rake height from the surface for the various measurements. Table 3 translates the cam-speed parameter $N$ into disturbance period $\tau$ and lists the various combinations of spanwise and streamwise periods that were studied. A number entered into table 3 identifies a figure in this paper in which the intermittency data may be found. An asterisk indicates that similar data are reported in Savaş (1979a). Parentheses indicate that unreported data exist for three or more stations in the form of preliminary plots.

A systematic method was needed for setting the hot-wire rake at a suitable distance from the plate surface. During some preliminary runs with the $12.2 \mathrm{~cm}$ cam, it became apparent that a disturbance frequency of $25 \mathrm{~Hz}(N=800)$ produced a very regular, coherent flow over the full length of the plate (see figure $11 a$ below). Some experimenting showed that a good rake position for this flow was one where the global intermittency (for unfiltered data averaged over all 24 wires) was about 0.4 . This value was therefore adopted as a target to be set at each station for $N=800$ for each cam, and the probe position was left unchanged for all other runs with the same cam. As an example of the experimental experience figure 6 shows analog signals obtained from two of the hot wires, with their associated intermittency signals, when the rake was at the most upstream station and the $12.2 \mathrm{~cm}$ cam was running at $N=1991$. 


\begin{tabular}{lcccccc} 
Station $\ldots$ & 4 & 8 & 12 & 16 & \multicolumn{1}{c}{20} & 24 \\
$x(\mathrm{~cm}) \ldots$ & 56.9 & 87.3 & 117.8 & 148.3 & 178.8 & 209.3 \\
Tape trip & 1.30 & 1.70 & 2.08 & 2.45 & 2.77 & 3.14 \\
12.2 cm cam & 0.63 & 1.36 & 1.76 & 2.18 & 2.52 & 2.89 \\
$9.1 \mathrm{~cm}$ cam & 0.73 & 1.36 & 1.78 & 2.18 & 2.52 & 2.89 \\
$6.1 \mathrm{~cm}$ cam & 0.89 & 1.43 & 1.86 & 2.25 & 2.57 & 2.95
\end{tabular}

TABLE 2. Distance of hot-wire rake from surface in centimetres for $N=800,\langle\gamma\rangle=0.4$.

\begin{tabular}{ccccc} 
& & \multicolumn{3}{c}{$\zeta(\mathrm{cm})$} \\
$N$ & $\tau(\mathrm{s})$ & 12.2 & 9.1 & 6.1 \\
4953 & 0.248 & () & () & $*$ \\
2867 & 0.143 & () & () & () \\
2389 & 0.119 & () & () & () \\
1991 & 0.100 & $*$ & $9 a$ & $*$ \\
1659 & 0.083 & $10 a$ & $*$ & $*$ \\
1382 & 0.069 & $*$ & $*$ & $9 b$ \\
1152 & 0.058 & $*$ & $10 b$ & $*$ \\
960 & 0.048 & $*$ & $*$ & $*$ \\
800 & 0.040 & $11 a$ & $*$ & $10 c$ \\
667 & 0.033 & $*$ & $*$ & $*$ \\
556 & 0.028 & $12 a$ & $11 b$ & $*$ \\
463 & 0.023 & $*$ & $*$ & $*$ \\
386 & 0.019 & $*$ & $12 b$ & $11 c$ \\
322 & 0.016 & - & () & () \\
268 & 0.013 & - & () & -
\end{tabular}

* See Savaş (1979a) for figure; ( ), preliminary plots exist.

TABLE 3. Scope of experiments. Numerical entries in last three columns correspond to figure numbers in the present paper.

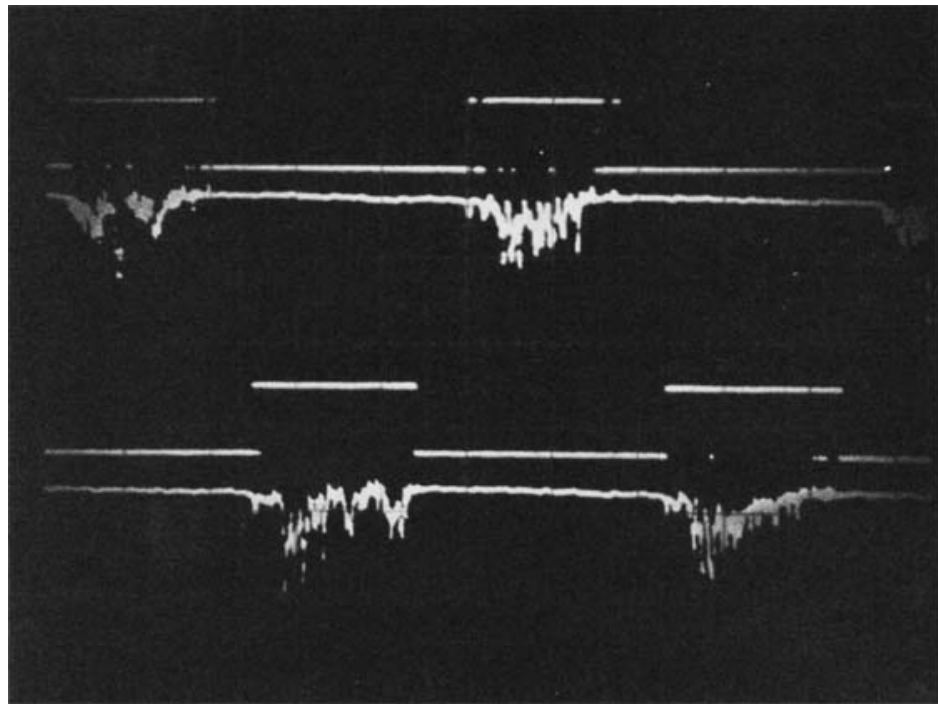

Figure 6. Example of hot-wire analog signals and associated intermittency signals in synthetic turbulent boundary layer. Flow is for $12.2 \mathrm{~cm}$ cam, $N=1991, x=56.9 \mathrm{~cm}, y=0.63 \mathrm{~cm}$ (run 190). Oscilloscope is triggered at index pulse. Upper traces: hot-wire $13(z=0.38 \mathrm{~cm})$. Lower traces: hot-wire $21(z=6.48 \mathrm{~cm})$. One camshaft revolution is shown, at sweep rate of $20 \mathrm{~ms} / \mathrm{div}$. 


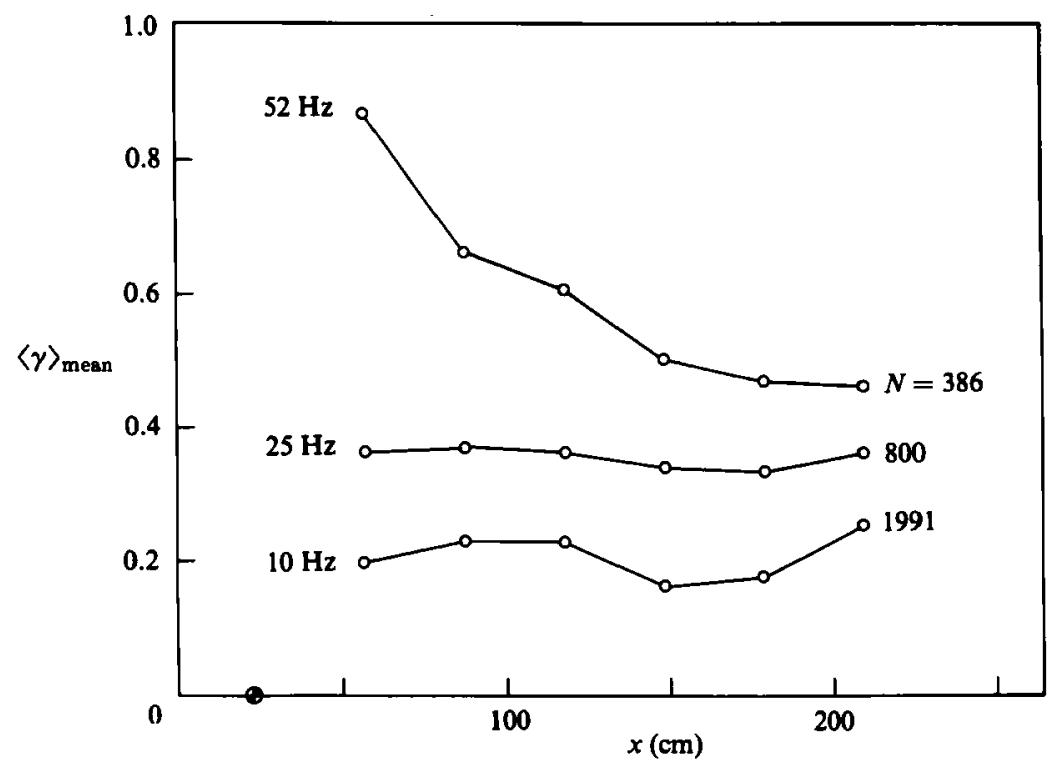

FIGURe 7. Variation of global intermittency as seen by rake for fixed cam speed but different rake stations. Data are for $12.2 \mathrm{~cm}$ cam.

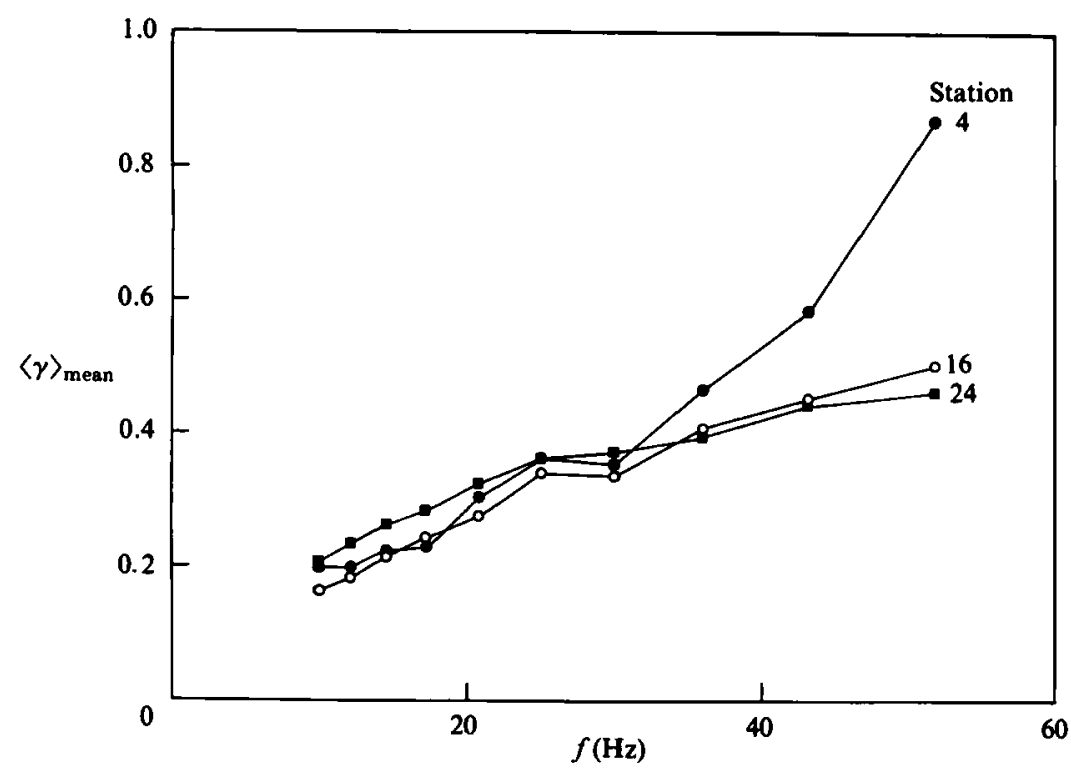

Figure 8. Variation of global intermittency as seen by rake for fixed rake position but different cam speeds. Data are for $12.2 \mathrm{~cm}$ cam. For stations, see table 2.

Because spot size and streamwise spacing change with frequency, and the apparent origin for spot growth also changes, one consequence of this scheme is that the global intermittency changes significantly with $x$ for fixed $N$ (for $N \neq 800$ ), as shown in figure 7 , and also changes with $N$ for fixed $x$, as shown in figure 8 . The values plotted in these figures are mean intermittency for filtered data, averaged over one revolution and one spanwise period.

Four families of flows from the present research are shown in figures 9-12. In each 


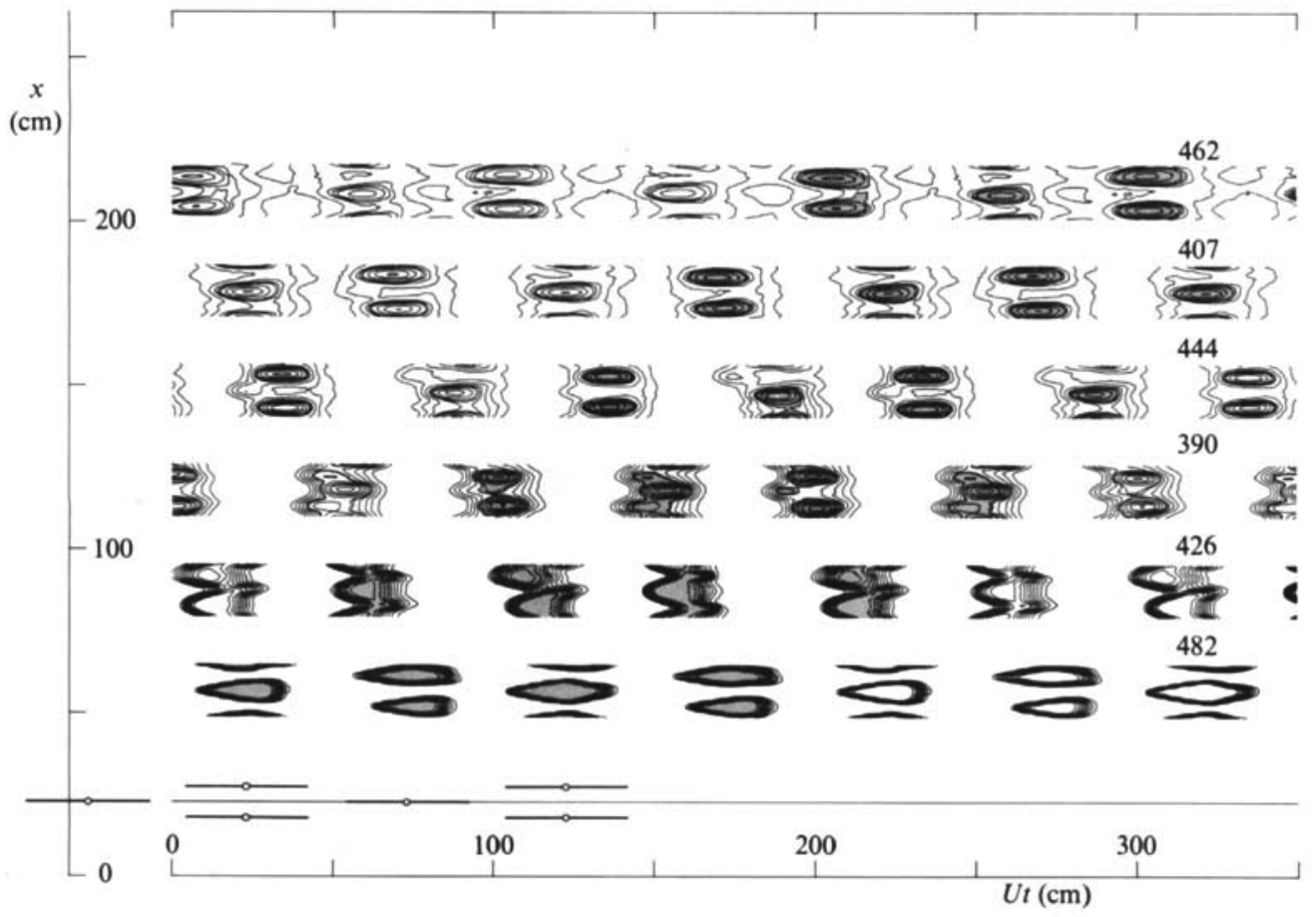

(a)

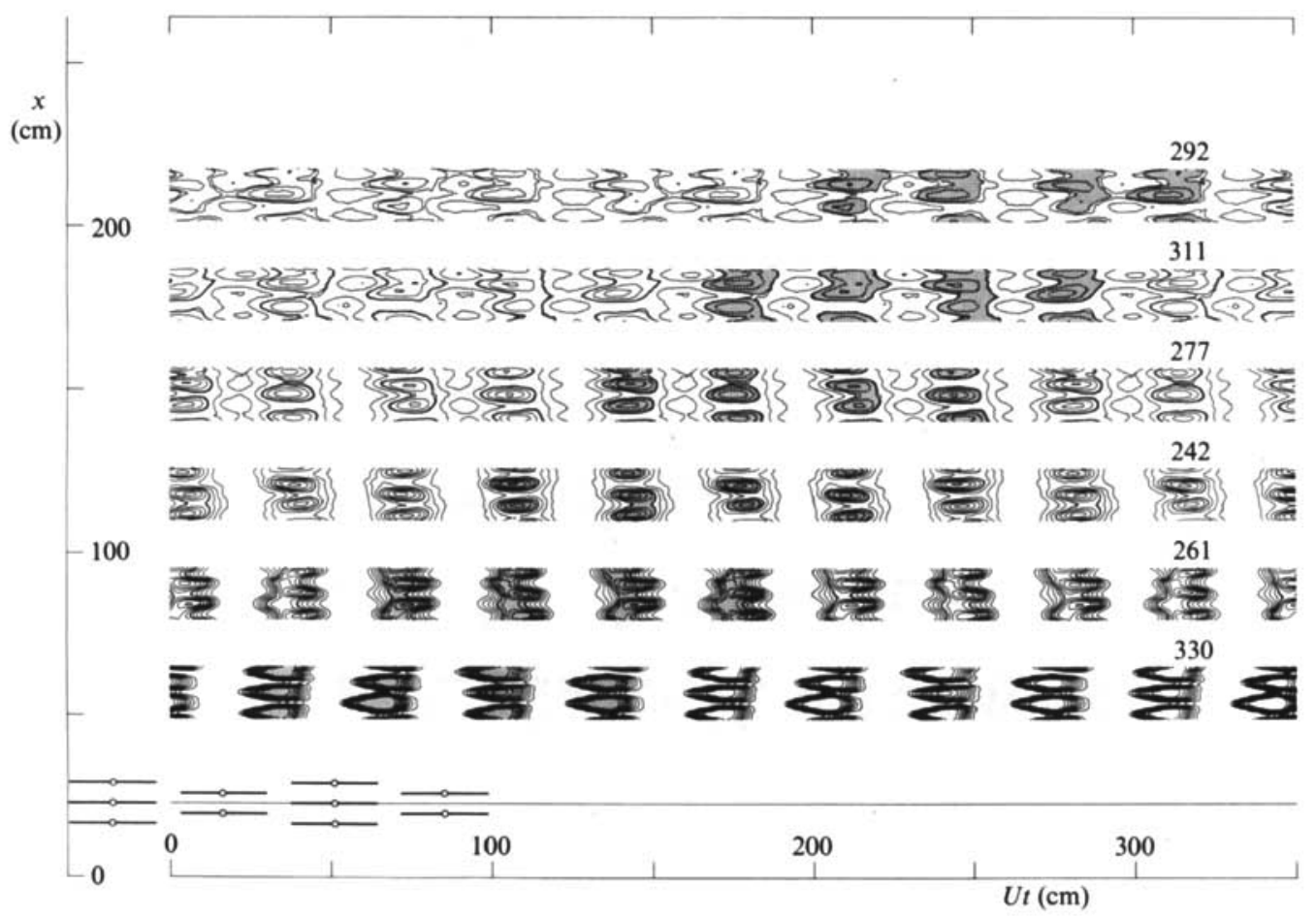

(b)

Figure 9. Intermittency in synthetic turbulent boundary layers with comparable values of $\zeta / u_{\infty} \tau \sim 0.09$. Contour interval is 0.1 . For rake distances from wall, see table $2 .(a) 9.1 \mathrm{~cm}$ cam, $N=1991, \zeta / u_{\infty} \tau=0.091$. Eddy transposition is complete at fourth station. (b) $6.1 \mathrm{~cm}$ cam, $N=1382, \zeta / u_{\infty} \tau=0.087$. Eddy transposition is complete at third station. 


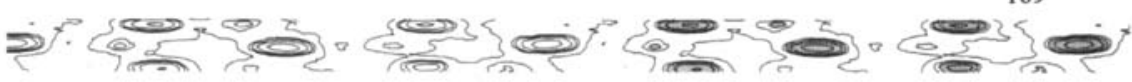

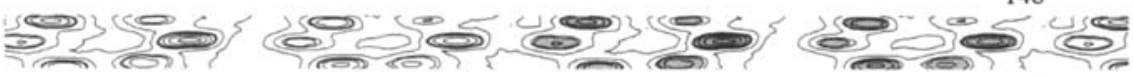

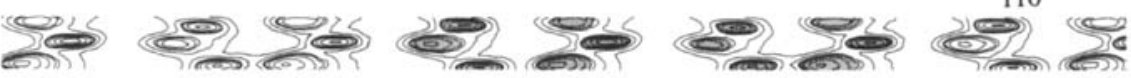
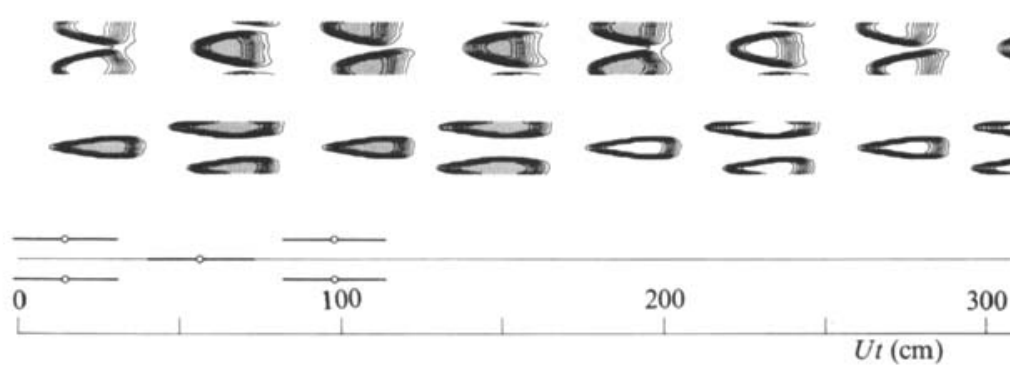

(a)

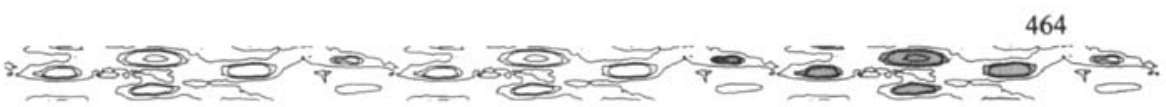

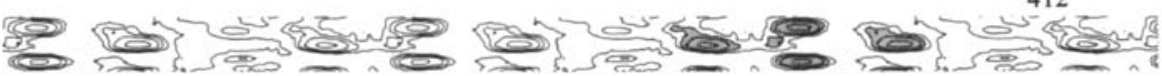

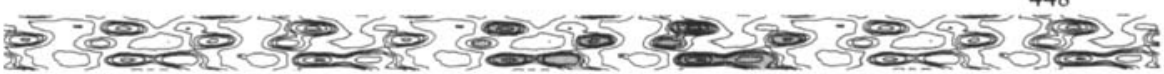

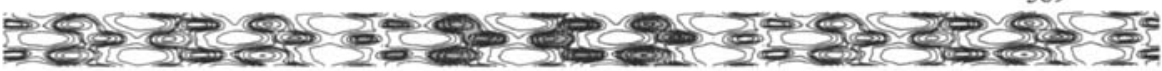

$-100-432$

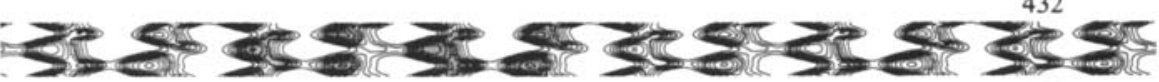

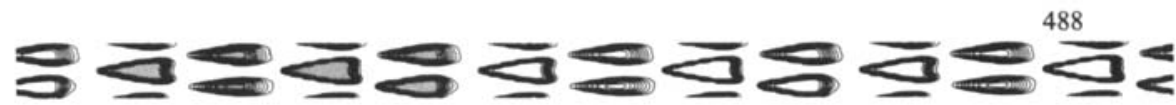

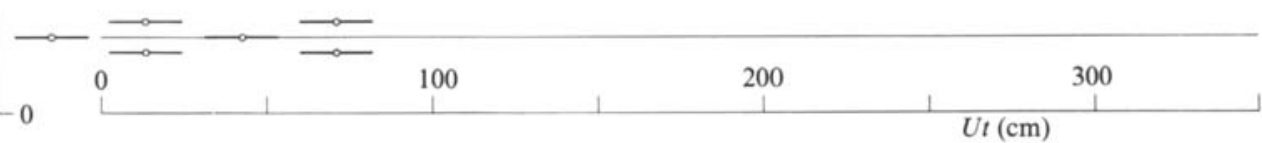

(b)

Figure $10(a)-(b)$. For caption see facing page. 


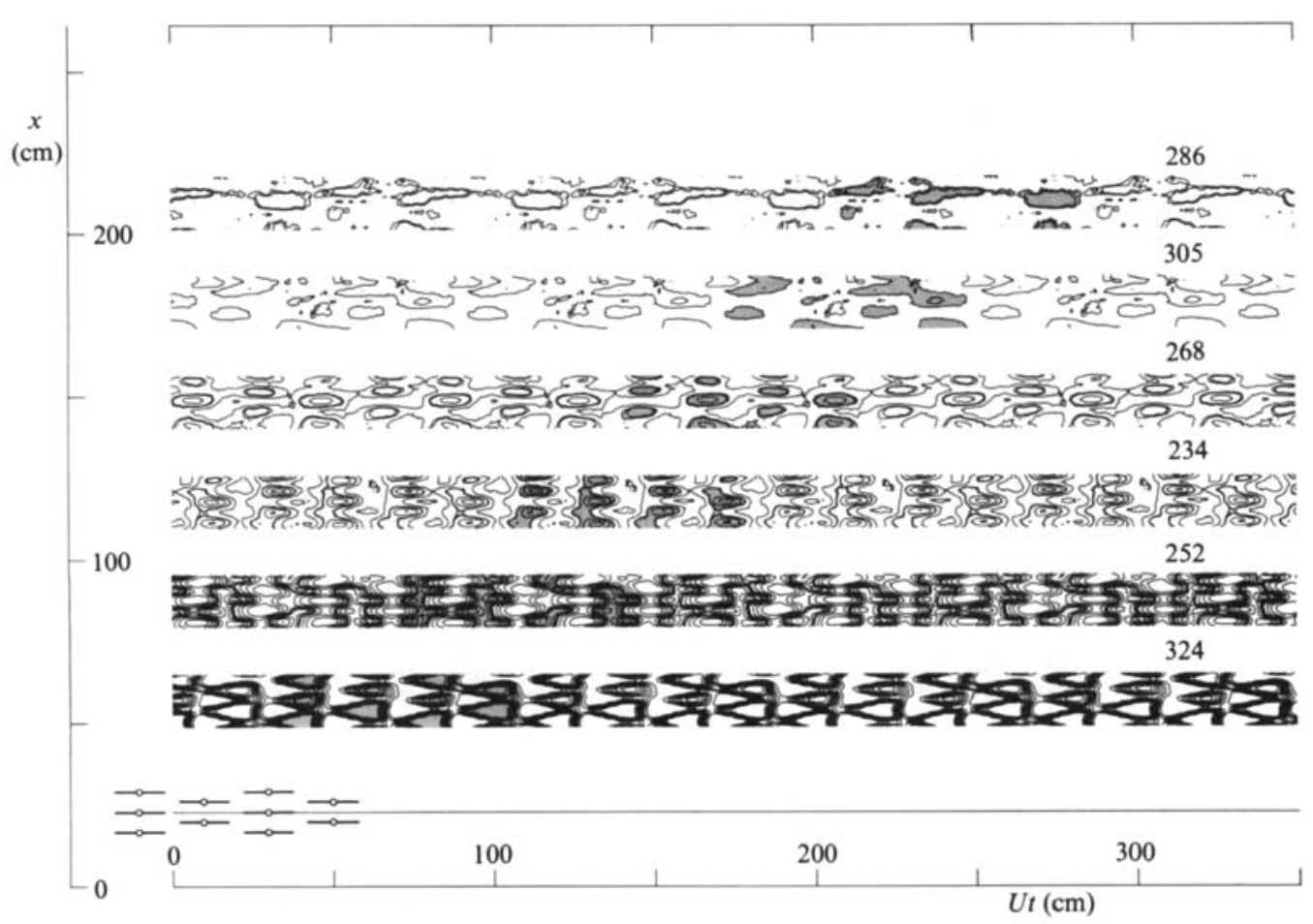

(c)

FIGURE 10. Intermittency in synthetic turbulent boundary layers with comparable values of $\zeta / u_{\infty} \tau \sim 0.15$. Contour interval is 0.1 . For rake distances from wall, see table 2 . (a) $12.2 \mathrm{~cm}$ cam, $N=1659, \zeta / u_{\infty} \tau=0.145$. Eddy transposition is not complete at sixth station. (b) $9.1 \mathrm{~cm}$ cam, $N=1152, \zeta / u_{\infty} \tau=0.157$. Eddy transposition is complete at fifth station. (c) $6.1 \mathrm{~cm}$ cam, $N=800$, $\zeta / u_{\infty} \tau=0.151$. Eddy transposition is complete at fourth station.

of these figures the ensemble-averaged intermittency $\langle\gamma\rangle$ is presented as a function of the three independent variables $x, z$ and $t$ (streamwise distance, spanwise distance, and time, or more properly phase, respectively). The main coordinates in each figure define the $(x, t)$-plane. The origin in time is the index pulse from the optical encoder on the cam-drive motor. At each $x$ corresponding to one of the six probe stations, a centred strip of data shows contours of constant ensemble-averaged intermittency in coordinates $(z, t)$; i.e. in coordinates representing a plan view of activity in a narrow strip symmetrical about the plate centreline at a fixed distance from the plate surface. The associated three-digit numbers are run numbers. These are filtered data, so that the two end wires are not used directly, and the effective width of the rake is $16.0 \mathrm{~cm}$. The contour interval in $\langle\gamma\rangle$ is 0.1 . The pattern in each strip is periodic with period $U t=2 U \tau$, where $\tau$ is the time required for half of a camshaft revolution. The figures therefore depict the downstream evolution of an average disturbance pattern. To assist in visualization the region inside the median contour is shaded for one particular pattern, which is generated beginning approximately at $t=0$ and is followed downstream. The solid horizontal lines and the small circles at the pin station indicate, respectively, the time interval during which the flow is disturbed and the moment when the pins are at top dead centre. Note that the coordinates $x, z$ and $U t$ are all in $\mathrm{cm}$ and have the same scale, so that the intermittency data show the 


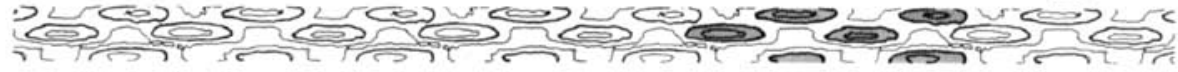

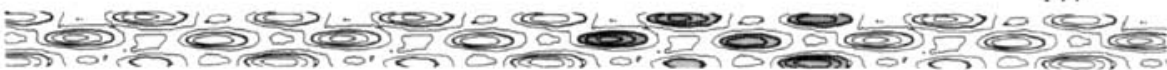

का

(c) 5 (2)
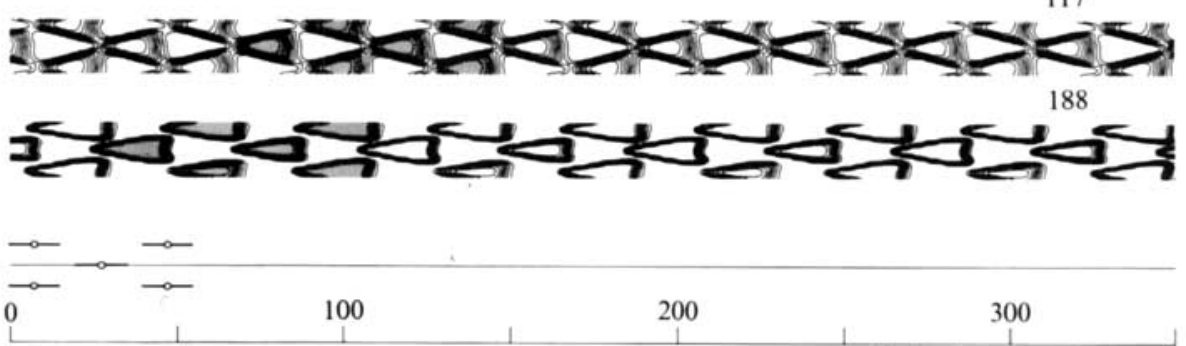

\section{0} 300

$U t(\mathrm{~cm})$

(a) D.

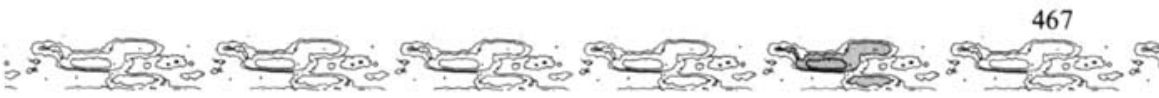
410

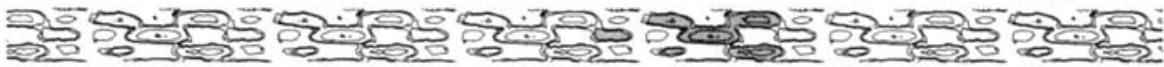
446

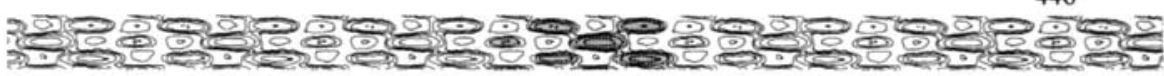

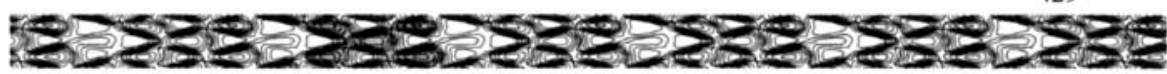

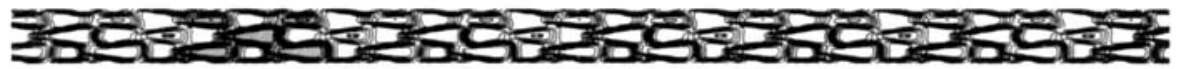

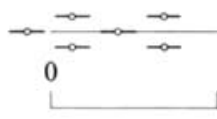

$$
100
$$

200

300

$U t(\mathrm{~cm})$

(b)

Fiaure $11(a)-(b)$. For caption see facing page. 


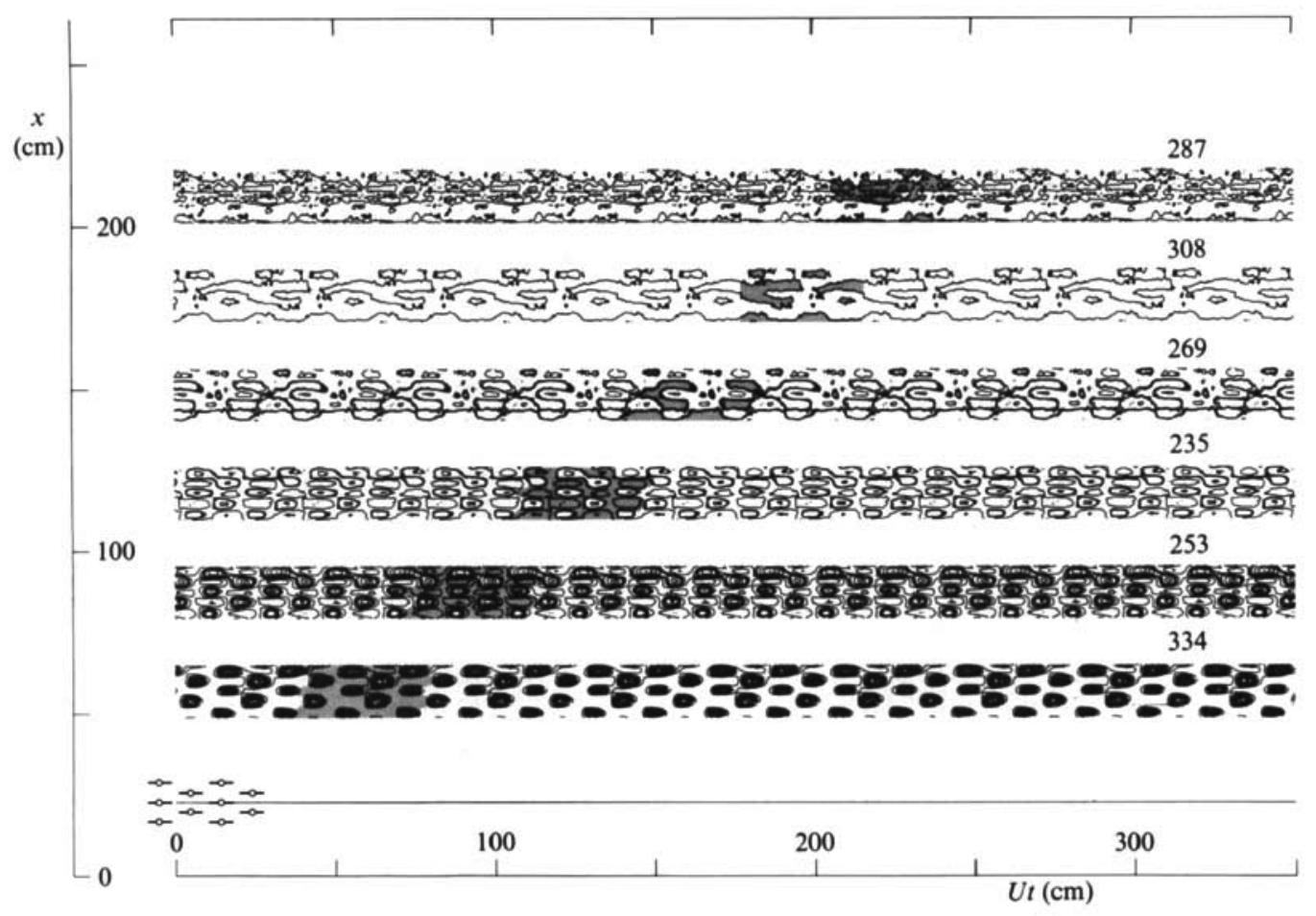

(c)

Figure 11. Intermittency in synthetic turbulent boundary layers with comparable values of $\zeta / u_{\infty} \tau \sim 0.31$. Contour interval is 0.1. For rake distances from wall, see table 2 . (a) $12.2 \mathrm{~cm}$ cam, $N=800, \zeta / u_{\infty} \tau=0.301$. (b) $9.1 \mathrm{~cm}$ cam, $N=556, \zeta / u_{\infty} \tau=0.325$. (c) $6.1 \mathrm{~cm} \mathrm{cam,} N=386$, $\zeta / u_{\infty} \tau=0.312$.

various flow patterns without distortion except for the small difference between celerity $c$ and reference velocity $U$, or more precisely free-stream velocity $u_{\infty}=1.013 U$.

\section{Results and discussion}

\subsection{General observations}

During the present study of synthetic turbulent boundary layers the two main parameters, pin spacing and cam speed, were varied over a substantial range, with the expectation that this range would include one or more synthetic flows deserving of eventual closer study. This expectation was realized in full.

Figures 9-12, taken as a whole, establish the important conclusion that the downstream large-eddy pattern develops directly from the original disturbance pattern, with an explicit correspondence between spots and large eddies. The data, beginning with figure 5, also confirm the photographic evidence in figure 1 and a related finding by Zilberman et al. (1977). There is a severe reduction in the growth rate of each spot, in both the spanwise and streamwise directions, as it moves downstream in a crowd of neighbouring spots. The growth rate normal to the surface, however, is almost unaffected (cf. table 2). The data do not support the conclusion by Elder (1960) thit overlapping spots grow independently of each other and can 


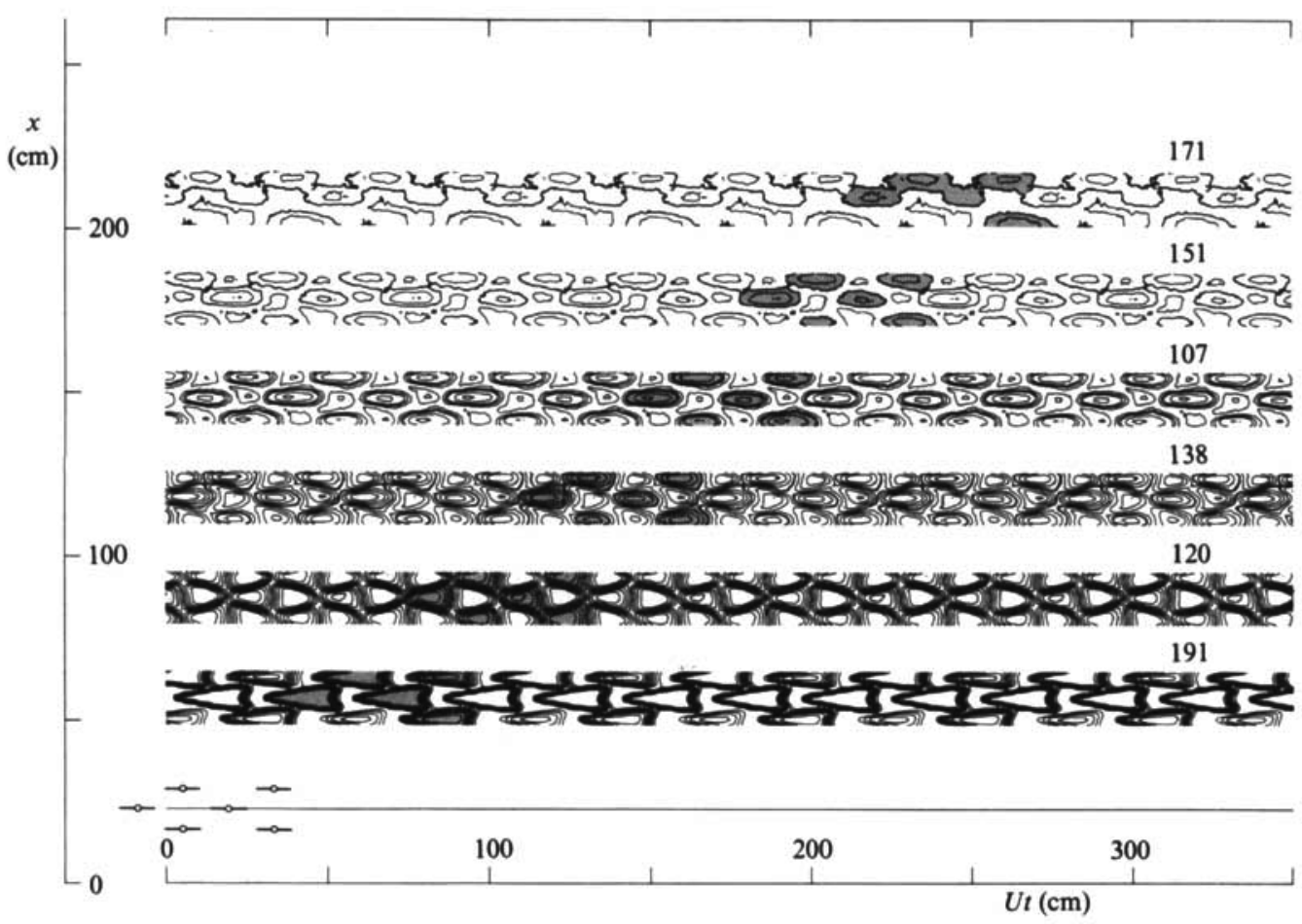

(a)

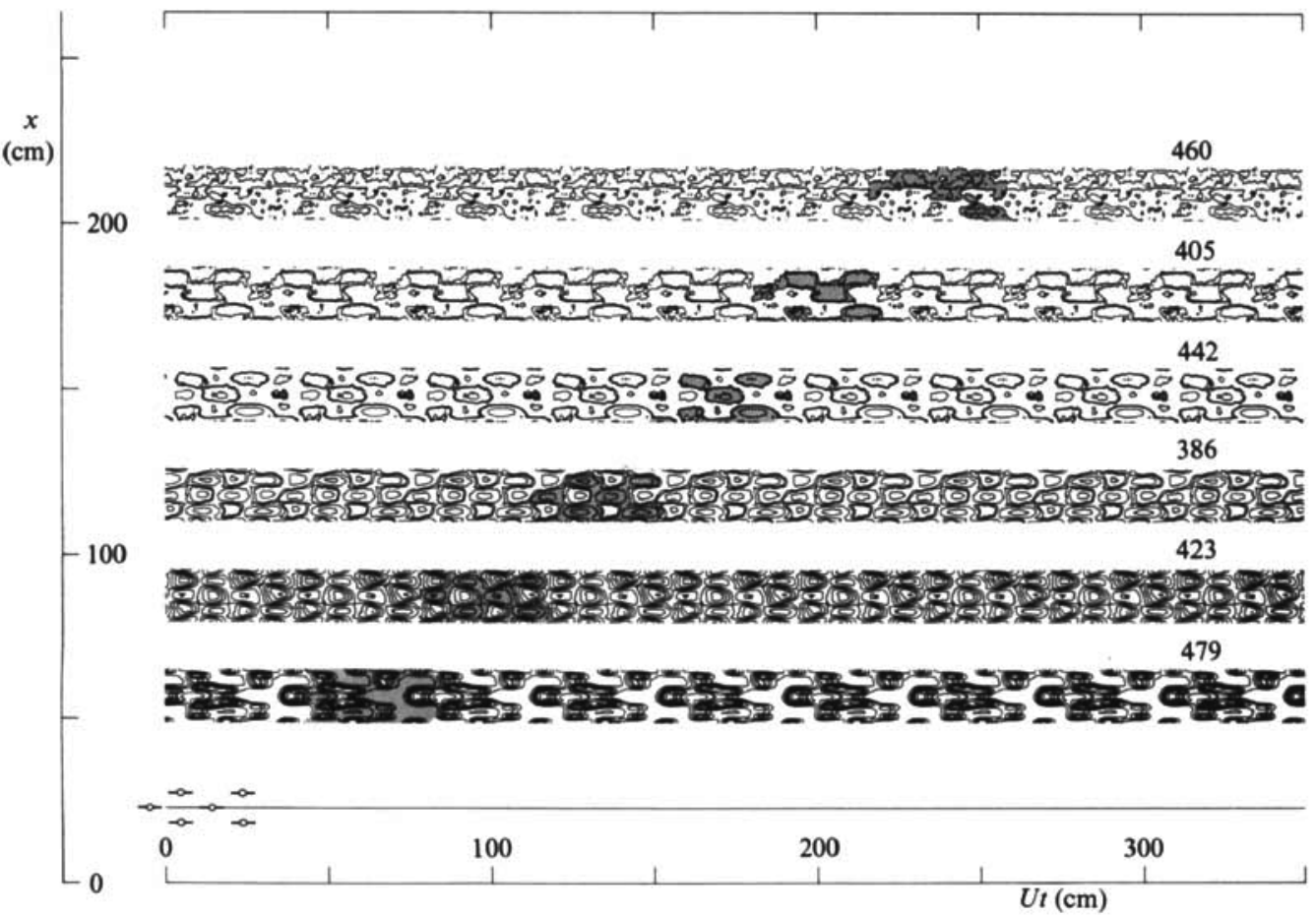

(b)

FigURE 12. Intermittency in synthetic turbulent boundary layers with comparable values of $\zeta / u_{\infty} \tau \sim 0.45$. Contour interval is 0.1 . For rake distances from wall, see table 2 . (a) $12.2 \mathrm{~cm} \mathrm{cam}$, $N=556, \zeta / u_{\infty} \tau=0.433$. (b) $9.1 \mathrm{~cm} \operatorname{cam}, N=386, \zeta / u_{\infty} \tau=0.468$. 
be treated by superposition. Elder studied only the case of two spots side by side, and then only at an $x$-Reynolds number of about $0.4 \times 10^{6}$. The present data deal with large arrays of spots and with the range of $x$-Reynolds numbers from $0.4 \times 10^{6}$ to $1.3 \times 10^{6}$.

Another important result concerns an interaction phenomenon which we propose to call eddy transposition. This phenomenon is conspicuous in figures 9 and 10. It involves the appearance and rapid growth of regions of new turbulence at the rear of the original spots and in the gaps between them. The original spots then decay and disappear. In the middle of the transposition process (for example, at the fourth station in figure $10 a$ ), the number of large eddies is twice the normal value, and these eddies form a honeycomb pattern of small hexagons with empty centres. When the transposition process is complete, the original hexagonal pattern is restored, but with a permanent phase shift. In most but not all cases, at least the early stages of the transposition process involve configurations having extensive regions near the wall that are free or almost free of turbulence. The phenomenon of eddy transposition is probably closely related to the discovery by Wygnanski, Haritonidis \& Kaplan (1979) of oblique regular wave packets, resembling Tollmien-Schlichting waves, in the laminar boundary layer near the trailing tips of a single turbulent spot. These wave packets were seen to break down and form new turbulent regions. It is likely, therefore, that eddy transposition should be classified as part of the transition process and should not be expected to be conspicuous in a fully turbulent flow. It is even possible that eddy transposition can occur more than once during the transition process.

A different question is raised by the disappearance of the original eddies in the late stages of transposition. Our present view is influenced by a suggestion made by Cantwell et al. (1978) regarding the process which supplies energy to a turbulent spot. Their evidence shows that free-stream fluid overtakes the spot and is entrained and mixed at the rear of the spot, where the surface friction is large (the large friction may be thought of as either a cause or an effect of the mixing and deceleration). It may be that the rear eddies shield the front ones during transposition and reduce their energy supply to such a level that they are obliged to decay and disappear. This conjecture, if it is correct, implies that a configuration with large eddies following in line, one behind the other, would not be stable. Hence a rectangular pattern would be a poor choice for a synthetic flow, whereas a hexagonal pattern would be a good one. We should acknowledge here that our original reasons for choosing a hexagonal pattern, following Coles \& Barker, were aesthetic rather than scientific. In any event, an experimental test of the conjecture is clearly feasible.

The rear of a turbulent spot is also a region of high surface pressure (see figure 28 of Savaş 1979a; also Mautner \& Van Atta 1982). Thomas \& Bull (1983) have recently identified the pressure signature of the large eddy in a fully developed turbulent boundary layer. Their figure 22 indicates a low-pressure region at the front followed by a high-pressure region at the rear. This finding also points towards a similarity between the fully developed turbulent boundary layer and a synthetic boundary layer constructed from individual spots.

\subsection{Celerity}

In any flow configuration where the large eddies remain intact as they move downstream, their celerity can be easily measured as the slope $U \mathrm{~d} x / \mathrm{d} U t$ in figures like 9-12. Such a measurement, corrected for the small difference between $U=1000 \mathrm{~cm} / \mathrm{s}$ and $u_{\infty}=1013 \mathrm{~cm} / \mathrm{s}$, and with strong emphasis on the data at the 


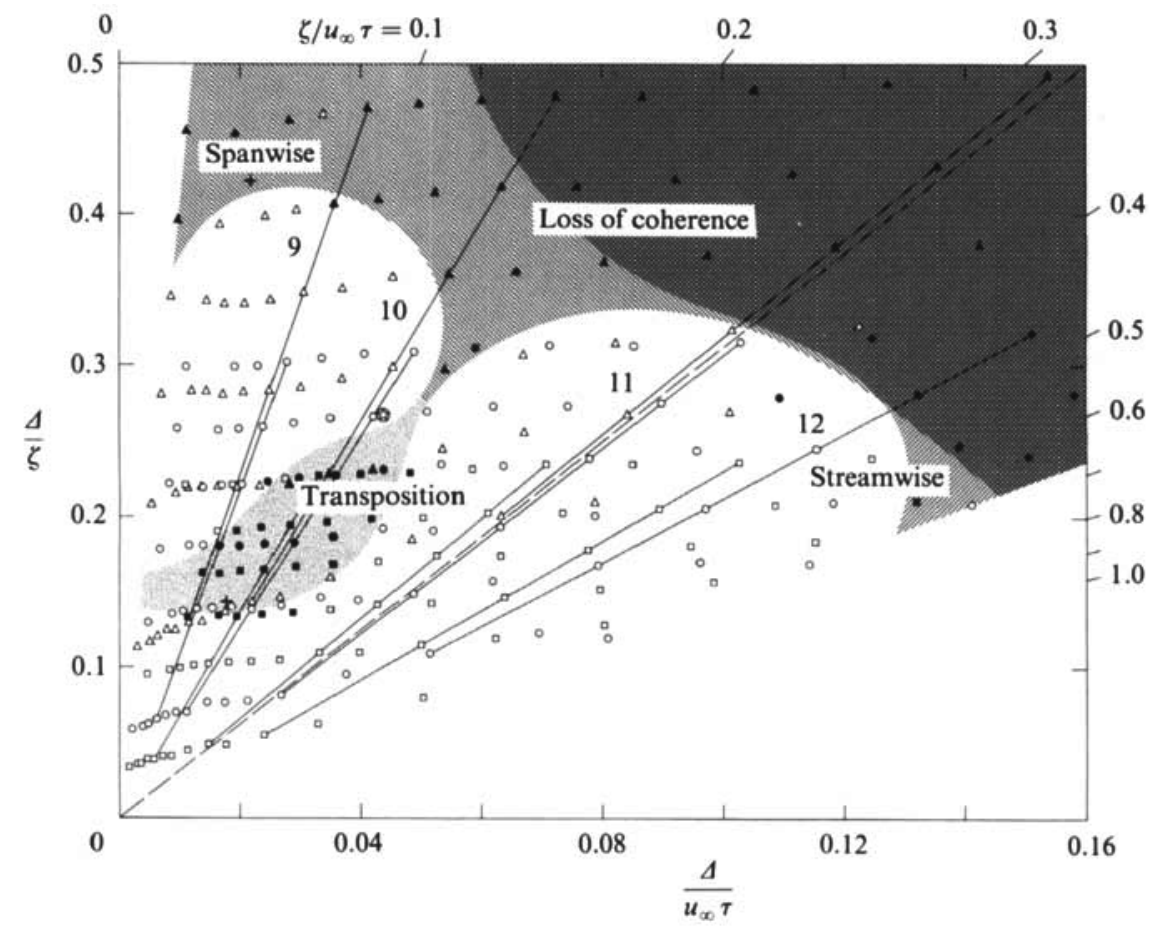

Fiaure 13. Coherence diagram for synthetic turbulent boundary layer. Open symbols, coherent pattern ; solid symbols and shading, loss of coherence (or transposition); $\square, 12.2 \mathrm{~cm}$ cam; $0,9.1 \mathrm{~cm}$ cam; $\triangle, 6.1 \mathrm{~cm}$ cam; labelled groups of lines, see figures $9-12$; dashed line, $\zeta / u_{\infty} \tau=0.32$; star, flow studied by Coles \& Barker (1975); + , flows studied by Chambers (1982).

more downstream stations, yields a constant celerity of $0.88( \pm 0.02) u_{\infty}$. The dispersion in this value is more a measure of experimental scatter than of any systematic dependence of celerity on either of the two scales of the hexagonal eddy pattern. The phenomenon of eddy transposition, in particular, affects the position but not the celerity of the large eddies. These observations diverge somewhat from the findings of Kovasznay, Kibens \& Blackwelder (1970) as presented in their figure 16. Their results indicate that the average celerity of the turbulent bulges in the outer region of a fully developed turbulent boundary layer is about $0.93 u_{\infty}$.

\subsection{Coherence diagram}

In figure 13, the present experimental results for different cams, different cam speeds, and different stations have been collected in one diagram to show the kinds of interactions and evolutions that can occur in a synthetic boundary layer. On the reasonable assumption that the flow is not much affected by viscosity, variables which suggest themselves for this purpose are the spanwise and streamwise periods normalized by some measure of the local layer thickness. Because the patterns vary from open and highly regular (figure $11 a$ ) to closed and nearly incoherent (figure 12b), it is not a simple matter to define a layer thickness consistently. The nearest available variable is the rake height $\Delta$, which was adjusted at each of the six stations to obtain a global unfiltered intermittency $\langle\gamma\rangle$ close to 0.40 for the particular cam speed $N=800$, and was left unchanged for other cam speeds. At each rake station an experimental value is also available for the derivative $\partial\langle\gamma\rangle / \partial y$ for $N=800$, as a byproduct of the adjustment process just mentioned. For each run, therefore, the rake 
height $\Delta$ has been corrected for other cam speeds by estimating the height corresponding to $\langle\gamma\rangle=\mathbf{0 . 4 0}$. The correction to $\Delta$ is appreciable for extreme values of cam speed and especially for the first one or two rake stations (see figures 7 and 8). On the basis of intermittency measurements by Klebanoff (1954) in a tripped bounday layer, it is reasonable, at least for synthetic flows with cam speeds that are not too high or too low, to make the rough tentative association $\Delta=0.8 \delta$, where $\Delta$ is the probe height at which $\langle\gamma\rangle=0.40$, and $\delta$ is the boundary-layer thickness according to some more conventional definition.

The corrected data are displayed in figure 13. This figure is the main product of the present research. The coordinates vary inversely with the two basic scales, which are taken to be $u_{\infty} \tau$ in the streamwise direction and $\zeta$ in the spanwise direction. Each of the 42 flows observed experimentally (table 3 ) is characterized by fixed values for these two scales and for their ratio $\zeta / u_{\infty} t$, with the local operating point moving radially away from the origin as the pattern proceeds downstream and $\Delta$ increases. Data for the three cams are denoted by three different symbols. The flows depicted in figures 9-12 are represented by four groups of continuous straight lines, with a figure number attached to each group for identification.

The solid symbols and the shaded areas in the upper part of figure 13 denote regions where there is substantial loss of coherence. The more complex boundary refers to the spanwise direction. To define this boundary, it is first necessary to define a quantitative measure for dispersion in the intermittency strips in figures 9-12 (and 32 other similar figures). The measure adopted here is that a symbol in figure 13 should be solid if the maximum value of the dimensionless spanwise derivative $|\partial\langle\gamma\rangle / \partial(z / \zeta)|$ in the corresponding strip in less than unity. Roughly speaking, a value less than about 2.5 already guarantees that full modulation between the limits 0 and 1 can no longer be expected for the ensemble-averaged intermittency surface $\langle\gamma\rangle(z, t)$ at a fixed distance from the wall. A second shaded area in the upper part of figure 13 denotes loss of coherence in the streamwise direction, the boundary here being defined by the condition that the dimensionless streamwise derivative $|\partial\langle\gamma\rangle / \partial(t / \tau)|$ is less than unity.

The two loss-of-coherence boundaries just described diverge from each other for large $\tau$ and for large $\zeta$. Given a doubly periodic pattern, flow in the unexplored triangle near the vertical axis must evolve towards the limiting case of the isolated two-dimensional spot. The limit is reached on the axis, provided that the distance from the disturbance station is less than $u_{\infty} \tau$ (if it is supposed, say, that the spot leading and trailing edges move at $u_{\infty}$ and $\frac{1}{2} u_{\infty}$, respectively). Similarly, flow in the unexplored triangle near the horizontal axis must evolve towards the limiting case of the isolated turbulent wedge, provided that the distance from the disturbance station is less than about $3 \zeta$ (if the wedge grows with a half-angle of $10^{\circ}$ ). In neither limit is the thickness $\Delta$ a useful parameter.

Another shaded area in the left centre of figure 13 denotes the region where eddy transposition occurs. This region is defined in part by a subjective classification of the intermittency strips. An objective criterion, met by the flows represented by solid symbols, is that the number of distinct turbulent islands or eddies should be locally larger than the number originally generated upstream. For this purpose, a turbulent island is recognized only if it has at least two closed intermittency contours. Several flows in the left part of figure 13 pass through the transposition region and emerge as displaced but still coherent patterns, as illustrated by the example in figure $10 a$. The suggestion that the transposition region extends indefinitely to the left near $\Delta / \zeta=0.15$ is somewhat tentative, but is consistent with the available data.

In the main body of figure 13 , the various boundaries coincide reasonably well for 
different cams. It follows that the scales $\Delta, \zeta$ and $u_{\infty} \tau$ are appropriate for characterizing synthetic flows in this region, while the scales $x$ and $\nu / u_{\infty}$ are not. On the other hand the free-stream velocity, the disturbance station, and the duty cycle for deployment of the disturbance mechanism were not varied during the present experiments. Because the layer thickness $\Delta$ at a fixed station is not very sensitive to the disturbance frequency, maximum spanwise coherence can be formally associated with a horizontal tangent at the spanwise loss-of-coherence boundary. The most coherent flow therefore occurs when the global parameter $\zeta / u_{\infty} \tau$ is about 0.25 . However, the trajectory then passes uncomfortably close to the transposition boundary. This observation, together with other considerations outlined in the next section, suggests that a more appropriate value might be $\zeta / u_{\infty} \tau=0.32$. The latter value is represented quantitatively by the dashed diagonal line in figure 13 and qualitatively by the flow patterns in figure 11. Flows that are well suited for study of transposition per se can be found in the range near $\zeta / u_{\infty} \tau=0.10$ (cf. figure 9 ). The least coherent flow, characterized by strong transposition followed by rapid loss of coherence, lies at about $\zeta / u_{\infty} \tau=0.18$.

The synthetic flow studied by Coles \& Barker can be placed in figure 13 if an estimate is made of the boundary-layer thickness. It appears that the scales for this flow were not well chosen, and that the measurements fall in the late-transposition region, as indicated by the star at the point $\Delta / \zeta=0.27, \Delta / u_{\infty} \tau=0.044$. The present indication that transposition occurred in this flow may account for the observation by Coles \& Barker that the region of ensemble-averaged velocity defect passed the probe with its blunt end forward, rather than its narrow end.

We pointed out in the introduction that a central and so far unresolved question in the present research is the degree of equivalence that can be expected between synthetic and natural flows, other things being equal. Three recent papers have provided useful evidence on this question, although in all cases the work was aimed at the different issue of possible drag reduction in synthetic flows. Chambers (1982) measured streamwise-velocity profiles in a number of flows in the transposition regime, with special attention to two cases for which $\zeta / u_{\infty} \tau=0.05$ and 0.13 . At the two points marked by crosses in figure 13 , the ensemble-averaged velocity signals at all levels in the layer were found to be strongly modulated at the forcing frequency, but the time-averaged profiles, and the inferred friction coefficients, were essentially normal for the Reynolds numbers of the experiments. The same null result was obtained in a second paper by Chambers (1985) which reports time-averaged measurements farther downstream in a flow with $\zeta / u_{\infty} \tau==0.02$. These latter data are off scale in figure 13, with $\Delta / \zeta$ in the range $0.6-2.5$ (an earlier stage of a closely similar flow is depicted in figure 33 of Savaş $1979 a$ and figure 6 of Coles \& Savaş 1979). Finally, Goodman (1985) constructed several synthetic flows whose disturbance patterns were rectangular rather than hexagonal and were usually of quite small scale in both directions. The Reynolds numbers were close to the lowest values for which fully turbulent flow can be defined, so that Goodman's work seems to be primarily a study of a special kind of tripping device. Profile measurements were made but are not reported. A modest reduction in surface friction was observed under some conditions. The data in question are far off scale in figure 13, inasmuch as the variables $\zeta / u_{\infty} \tau, \Delta / \zeta$ and $\Delta / u_{\infty} \tau$ were all of order unity. None of these three papers discusses the question of structure. 


\subsection{Scales}

It remains to reconsider the question of scales mentioned in the Introduction. The correlation measurements made in a tripped boundary layer by Kovasznay et al. (1970) revealed an average large eddy about $1.2 \delta$ wide and $2.5 \delta$ long at the half-intermittency level. Such an eddy might command an area at most about $2 \delta$ wide and $4 \delta$ long at the wall. Other investigators have used counting methods to measure the frequency of various events which are believed to be related to the large-scale motion. For example, Kovasznay et al. counted interface crossings and found a maximum frequency $u_{\infty} / f \delta$ of about 1.3 at the half-intermittency level. This value was later confirmed by Chen \& Blackwelder (1978), who used heat as a passive contaminant and counted temperature-interface crossings. Various results obtained from the position of zero crossings in time correlations have been summarized, with new data at higher Reynolds numbers, by Badri Narayanan \& Marvin (1978). They recommend the value $u_{\infty} \tau / \delta=5$ to 6 . A recent survey by Fleischmann \& Wallace (1984) suggests a value $u_{\infty} \tau / \delta$ in the range $2.5-4.0$, if sublayer measurements are excepted. These experimental estimates of scale give values which are at variance with each other and are all appreciably smaller than the scales that characterize the most coherent region in figure 13. Note, however, that the experimental methods in question, if they were to be applied at random spanwise position in the synthetic flows depicted in figures 9-12, might be biased towards values corresponding to a time interval $\frac{1}{2} \tau$ rather than $\tau$.

We now propose a conceptual estimate based on a return to the idea, discussed in the Introduction, that the large eddy can be explicitly identified with the turbulent spot. In dye photographs of spots (see figure 1 above and figure 4 of Cantwell et al. 1978) the vortex position is usually quite well defined by a strong concentration of dye. The included angle between the two legs of the vortex in a plan view is about $40^{\circ}$. The same angle can be inferred from the angle of the dye concentrations in regions of transverse contamination, as well as from the aluminium-flake photographs in Cantwell et al. (see also Gad-el-Hak et al. 1981). Consider, therefore, the hexagonal pattern shown in figure 14, in which the diagonal lines represent vortex cores aligned in a regular way. The geometry of the sketch implies $\tan \frac{1}{2} \theta=\zeta / c \tau$. For $\theta=40^{\circ}$ and $c=0.88 u_{\infty}$, it follows that $\zeta / u_{\infty} \tau=0.32$. This condition is represented in figure 13 by the dashed line. For $u_{\infty}=1013 \mathrm{~cm} / \mathrm{s}$ and for $\zeta=12.2,9.1$ and $6.1 \mathrm{~cm}$, the appropriate values for $\tau$ are $0.038,0.028$ and $0.019 \mathrm{~s}$. The corresponding flows, according to table 3 , are those described by figure 11. These flows enter the loss-of-coherence region in figure 13 at about $\zeta / \Delta=3.2, U \tau / \Delta=10$. If $\Delta / \delta=0.8$, therefore, the flow in figure 15 should be marginally stable when

$$
\frac{\zeta}{\delta} \approx 2.5, \quad \frac{u_{\infty} T}{\delta} \approx 8
$$

Both values are close to the scales reported by Zilberman et al. (1977) and Haritonidis et al. (1977) for a spot immersed in a natural (i.e. tripped) turbulent boundary layer.

In summary, a combination of scales has been specified for constructing a synthetic turbulent boundary layer suitable for work on the signature problem discussed in the introduction. To operate in a marginal state at a given station and a given speed, it is only necessary to know (or guess) the layer thickness. The required cam spacing and cam speed then follow from the numerical estimates just derived. 


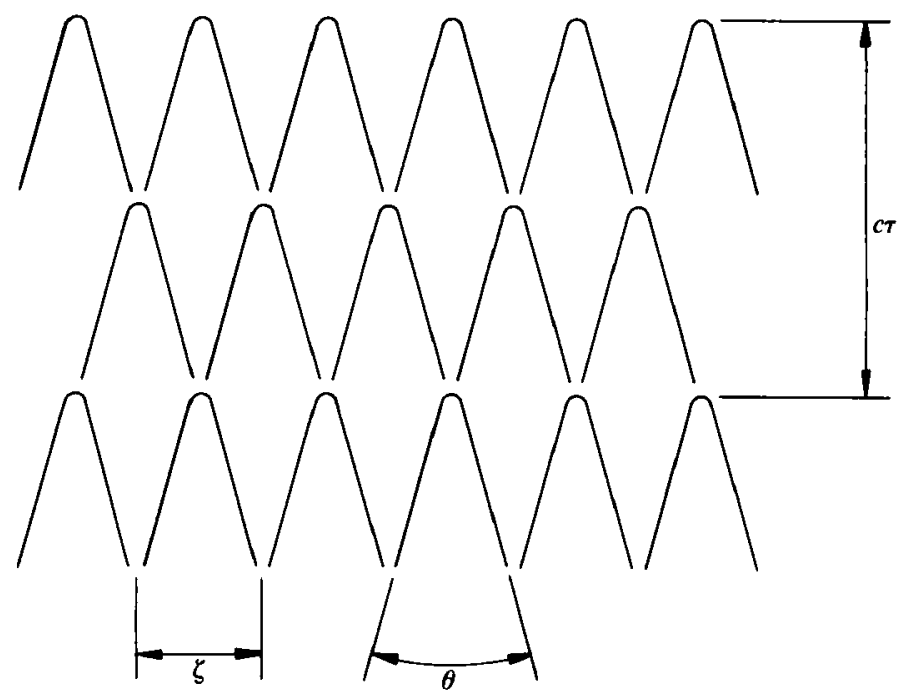

Figure 14. Postulated structure of synthetic turbulent boundary layer.

\subsection{Growth mechanism}

What is missing from the measurements so far is any quantitative measure of the dispersion or loss of coherence which is observed to occur as the synthetic flows move downstream. It is reasonable to suppose that this loss of coherence is associated with an insupportable geometrical evolution of the three-dimensional large-eddy structure as a result of entrainment. As a synthetic flow develops in the coherent region the spanwise and streamwise scales do not change, but the thickness increases almost linearly with time or distance. Eventually a phenomenon like the vortex pairing or coalescence first observed in the plane mixing layer by Brown \& Roshko (1974) and Winant \& Browand (1974) must occur. To fix the ideas, suppose that eddies in the boundary layer coalesce sufficiently rapidly for the volume of turbulent fluid not to change. That the volume of turbulent fluid is almost constant during pairing in the mixing layer has been established by computer analysis of high-speed shadowgraph movies taken by L. Bernal in the Brown-Roshko apparatus at GALCIT (Hernan \& Jimenez 1982). During a coalescence process in the present flow the spanwise and streamwise scales should then increase by a factor of two, while the thickness remains essentially unchanged. The main effect of coalescence must, therefore, be to reduce the relative thickness of the large eddies, and the situation should remain stable until the thickness has again grown by a factor of two. This argument implies that the most comfortable synthetic eddy for a given combination of spanwise and streamwise scales is one for which the thickness $\delta$ is about $40 \%$ less than the value at which loss of coherence becomes apparent in figure 13. This conclusion should be taken into account in any selection of scales for a synthetic boundary layer, as should the likely proposition, in view of the doubly periodic geometry in figure 14, that coalescence should occur more naturally for vortex fours than for vortex pairs.

It is even possible to speculate about the properties of a hypothetical synthetic flow so perfectly constructed that loss of coherence is itself a deterministic process. What should happen, once the flow reaches the boundary of the shaded region in figure 13, deserves to be called planned coalescence. The trajectory should reverse direction momentarily and then resume its normal evolution with new and larger scales. Since 
there is considerable design freedom in the method used for generating disturbances during the present research, such an experiment may be within reach.

The research described in this paper was supported by the National Science Foundation under Grants ENG 75-03694 and ENG 77-23541. Ö. Savaş gratefully acknowledges the Fellowship provided by the Scientific and Technical Research Council of Turkey (TUBITAK) during his stay at GALCIT.

\section{REFERENCES}

Badri Narayanan, M. A. \& Marvin, J. G. 1978 On the period of the coherent structure in boundary layers at large Reynolds numbers. In Coherent Structure of Turbulent Boundary Layers (ed. C.R. Smith \& D. E. Abbott), pp. 380-385. Lehigh University.

Brown, G. L. \& Roshko, A. 1974 On density effects and large structure in turbulent mixing layers. J. Fluid Mech. 64, 775-816.

CANTwell, B. J. 1975 A flying hot wire study of the turbulent near wake of a circular cylinder at a Reynolds number of 140,000 . Ph.D. thesis, California Institute of Technology.

Cantwell, B. J. 1981 Organized motion in turbulent flow. Ann. Rev. Fluid Mech. 13, 457-515.

CANTwell, B. \& Coles, D. 1983 An experimental study of entrainment and transport in the turbulent near wake of a circular cylinder. J. Fluid Mech. 136, 321-374.

Cantwell, B., Coles, D. \& Dimotakis, P. 1978 Structure and entrainment in the plane of symmetry of a turbulent spot. J. Fluid Mech. 87, 641-672.

Chambers, F. W. 1982 Preliminary measurements of a synthetic turbulent boundary layer. Lockheed-Georgia Co., Rep. LG82RR0009.

Chambers, F. W. 1985 Synthetically generated turbulent boundary layer development and structure. AIAA Paper 85-0534.

Chen, C.-H. P. \& BLAckwelder, R. F. 1978 Large-scale motion in a turbulent boundary layer: a study using temperature contamination. J. Fluid Mech. 89, 1-31.

Coles, D. \& BARKer, S. J. 1975 Some remarks on a synthetic turbulent boundary layer. In Turbulent Mixing in Nonreactive and Reactive Flows (ed. S. N. B. Murthy), pp. 285-292. Plenum.

Coles, D. \& SAvAş, Ö. 1979 Interactions for regular patterns of turbulent spots in a laminar boundary layer. In Proc. IUTAM Symp. on Laminar-Turbulent Transition, pp. 277-287. Springer-Verlag.

ELDER, J. W. 1960 An experimental investigation of turbulent spots and breakdown to turbulence. J. Fluid Mech. 9, 235-246.

Emmons, H. W. 1951 The laminar-turbulent transition in a boundary layer. Part I. J. Aero. Sci. $18,490-498$.

Fleischmane, S. T. \& Wallace, J. M. 1984 Mean streamwise spacing of organized structures in transitional and developed bounded turbulent flows. AIAA J. 22, 766-769.

Gad-el-Hak, M., Blackwelder, R. F. \& Riley, J. J. 1981 On the growth of turbulent regions in laminar boundary layers. J. Fluid Mech. 110, 73-95.

Goodman, W. L. 1985 Emmons spot forcing for turbulent drag reduction. AIAA J. 23, 155-157.

Haritonidis, J. H., Kaplan, R. E. \& Wygnanski, I. 1977 Interaction of a turbulent spot with a turbulent boundary layer. In Structure and Mechanisms of Turbulence I (ed. H. Fiedler), Lecture Notes in Physics No. 75, Springer-Verlag, 234-247.

Hernan, M. A. \& Jimenez, J. 1982 Computer analysis of a high-speed film of the plane turbulent mixing layer. J. Fluid Mech. 119, 323-345.

Itsweire, E. C. \& VAn ATta, C. W. 1984 An experimental investigation of coherent substructures associated with turbulent spots in a laminar boundary layer. J. Fluid Mech. 148, 319-348.

KLebanoff, P. S. 1954 Characteristics of turbulence in a boundary layer with zero pressure gradient. NACA Tech. Note 3178; also NACA Tech. Rep. 1247, 1955.

Kovasznay, L. S. G., Kibens, V. \& Blackwelder, R. F. 1970 Large-scale motion in the intermittent region of a turbulent boundary layer. J. Fluid Mech. 41, 283-325. 
MatsuI, T. 1979 Visualization of turbulent spots in the boundary layer along a flat plate in a water flow. In Proc. IUTAM Symp. on Laminar-Turbulent Transition, pp. 288-296. Springer-Verlag.

Mautner, T. S. \& VAN AtTa, C. W. 1982 An experimental study of the wall-pressure field associated with a turbulent spot in a laminar boundary layer. J. Fluid Mech. 118, 59-77.

SAVAş, Ö. 1979a Some measurements in synthetic turbulent boundary layers. Ph.D. thesis, California Institute of Technology.

SAVAŞ, Ö. $1979 b$ Some measurements in synthetic turbulent boundary layers. In AGARD CP 271, Turbulent Boundary Layers-Experiments, Theory and Modeling, Paper No. 23.

Schubauer, G. B. \& Klebanoff, P. S. 1955 Contributions on the mechanics of boundary-layer transition. NACA Tech. Note 3489; also NACA Tech. Rep. 1289, 1956.

Tномаs, A.S. W. \& BuLL, M. K. 1983 On the role of wall-pressure fluctuations in deterministic motions in the turbulent boundary layer. J. Fluid Mech. 128, 283-322.

Winant, C. D. \& Browand, F. K. 1974 Vortex puiring: the mechanism of turbulent mixing-layer growth at moderate Reynolds number. J. Fluid Mech. 63, 237-255.

Wygnanski, I. 1978 On the possible relationship between the transition process and the large coherent structures in turbulent boundary layers. In Coherent Structure of Turbulent Boundary Layers (ed. C. R. Smith \& D. E. Abbott), pp. 168-193. Lehigh University.

Wyananski, I., Haritonidis, J. H. \& Kaplan, R. E. 1979 On a Tollmien-Schlichting wave packet produced by a turbulent spot. J. Fluid Mech. 92, 505-528.

Wygnanski, I., Sokolov, M. \& Friedman, D. 1976 On a turbulent 'spot' in a laminar boundary layer. J. Fluid Mech. 78, 785-819.

Wygnanski, I., Zilberman, M. \& Haritonidis, J. H. 1982 On the spreading of a turbulent spot in the absence of a pressure gradient. J. Fluid Mech. 123, 69-90.

Zilberman, M., Wygnanski, I. \& Kaplan, R. E. 1977 Transitional boundary layer spot in a fully turbulent environment. Phys. Fluids Suppl. 20, S258-S271. 